\title{
Wave Propagation in Periodic Buckled Beams. Part I: Analytical Models and Numerical Simulations
}

\author{
Florian Maurin ${ }^{\mathrm{a}, *}$, Alessandro Spadoni ${ }^{\mathrm{a}}$ \\ ${ }^{a}$ Institute of Mechanical Engineering \\ École Polytechnique Fédérale de Lausanne (EPFL) \\ 1015 Lausanne, Switzerland
}

\begin{abstract}
Periodic buckled beams possess a geometrically nonlinear, load-deformation relationship and intrinsic length scales such that stable, nonlinear waves are possible. Modeling buckled beams as a chain of masses and nonlinear springs which account for transverse and coupling effects, homogenization of the discretized system leads to the Boussinesq equation. Since the sign of the dispersive and nonlinear terms depends on the level of buckling and support type (guided or pinned), compressive supersonic, rarefaction supersonic, compressive subsonic and rarefaction subsonic solitary waves are predicted, and their existence is validated using finite element simulations of the structure. Large dynamic deformations, which cannot be approximated with a polynomial of degree two, lead to strongly nonlinear equations for which closed-form solutions are proposed.

Keywords: periodic buckled beams, compressive \rarefaction supersonic \subsonic solitary waves, Boussinesq equation, strongly nonlinear wave equation.
\end{abstract}

\section{Introduction}

Certain buckled structures are periodic media which possess a geometrically (physically) nonlinear, load-deformation relationship and dispersive sources such that solitary waves are possible [1]. They differ from geometrically discrete nonlinear mechanical systems hosting

*Corresponding author: florian.maurin@gmail.com

Preprint submitted to Elsevier

April 17, 2016

(C) 2016. This manuscript version is made available under the Elsevier user license http://www.elsevier.com/open-access/userlicense/1.0/ 
solitary waves as nonlinear penduli [2,3], woodpile periodic structures [4], tensegrity structures [5, 6], origami-based metamaterials [7], complex lattices [8-10], and granular media $[11,12]$ in the sense that they are continuous. Buckled structures are also different from continuous systems for which large deformations induce material nonlinearities modeled by the standard continuum theory, incorporating characteristic material lengths $[8,13,14]$ or having reduced dimensions as plates and rods [3, 15-20].

Periodic buckling is encountered in various systems such as the cooling of thin film fixed to a substrate of different thermal-expansion coefficient [21, 22], stretched membranes [23], compressed cylinders [24-26], sandwich panels [27, 28], reinforced plates [29], lattices [3032 or beams resting on elastic foundation [21, 33-35]. In the present paper, the emphasis is placed on buckling by compression of a slender beam where the elastic foundation is replaced by guided or pinned supports as shown in Figs. 1a-b. Note that in [36, 37], wave propagation in undulated beams are also investigated, but the structure are originally curved (no buckling) such that no supports are required for the stability and the medium is linear for small deformations.

a)

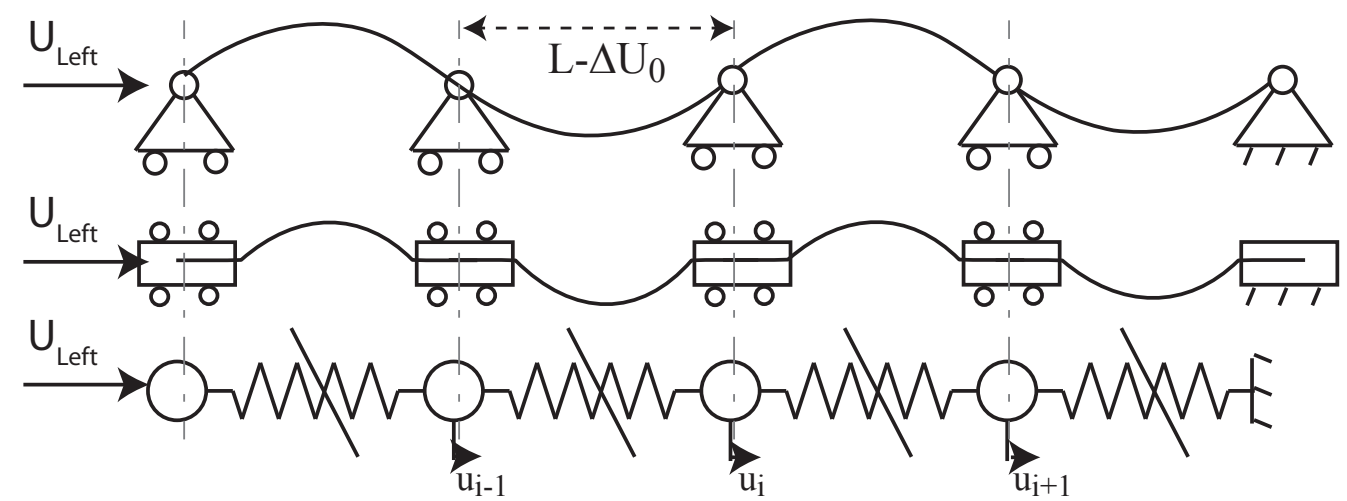

Figure 1: Guided (only axial displacement allowed) (a) and pinned (both axial displacement and rotation free) (b) support configurations composed of $p=4$ wavelengths and modeled by a chain of masses and nonlinear springs (c).

In a previous paper [1], and summarized in Sec. 2.1, we have shown that for a buckled beam described by a chain of masses and nonlinear springs (Fig. 1c), only considering dispersion due to periodicity, homogenization of the momentum equation leads to the Boussinesq 
equation with supersonic solitons as solution. However, although finite element (FE) simulations were in agreement with the supersonic soliton model for a highly-compressed beam, for small and moderate buckling level, a front, characteristic of subsonic nonlinear media, was present; this is in contradiction with our previous model. To this end, we have studied relevant dispersive sources using a semi-analytical dispersion relation [36], a method based on Bloch theorem and a transfer-matrix approach leading to an explicit power series of the wave number and frequency. In addition to periodicity, transverse inertia and displacementrotation coupling are founded important dispersion sources and dispersion analyses is summarized in Sec. 2.2.

. The homogenized model and dispersion analyses are summarized in Secs. 2.1 and 2.2 respectively.

It is the goal of the present paper to update Boussinesq models including the aforementioned additional dispersive sources, which vary according to pre-compression level and support type, and as it will be shown in Sec. 2.3, four type of solutions are present, namely compressive supersonic, rarefaction (tensile) supersonic, compressive subsonic and rarefaction subsonic solitary waves.

However, for nonlinear wave propagation with large amplitude or small pre-compression, the Boussinesq equation based on a local approximation of the load-displacement by a second degree polynomial is no longer valid. To this end, Sec. 2.4 propose two strongly nonlinear models, one based on a power-law nonlinearity similar to the one used for wave propagation in pre-compressed granular media, and an other one based on the exact load-displacement curve [11]. Existing solution techniques are extended here to account for the additional dispersion sources. Finite-element (FE) models and parameters are given in Sec. 3 followed in Sec. 4 by numerical simulations used to validate the derived homogenized models. Discussions and conclusions follow.

This paper, the first part of a series of two, concerns analytical models and numerical simulations for validation purposes and is followed by a second paper focused on experimental validation [38]. 


\section{Solitary waves in Buckled Structures}

The goal of the present paper is to generalize, update, and improve our previous models [1] used to describe wave propagation in one-dimensional (1D) buckled beams (Figs. 1a-b), and we start with a short review of previous work.

It is assumed for simplicity a slender beam free of shear, with linear-elastic material behavior and constant cross section parameters such that the cross-sectional area is denoted $A$, the area-moment of inertia $I_{z}$, the Young's modulus $E$, and the density $\rho$. In order to ensure stability of the $p$ buckled periodic wavelengths, $p+1$ supports, equally spaced by a distance $L$ are used (Figs. 1a-b). Although only pinned-supports were considered in [1], the derivation is generalized here to both guided and pinned supports (Figs. 1a-b) since dispersion depends on the support type [36].

\subsection{Solitary waves in buckled structures accounting for axial effects only}

Wave propagation is limited to the low-frequency regime treating deformations as quasistatic, and neglecting any phase lags between loads and deformations (see Appendix A for an a-posteriori justification). In this regard, wave propagation is described by the axial displacement of the supports, decomposed into $U\{t, x\}=U_{0}\{x\}+u\{t, x\}$ where $U_{0}\{x\}$ is the initial displacement due to pre-compression, $u\{t, x\}$ is the finite displacement increment in the buckled configuration, and $t$ and $x$ denote time and space, omitted hereafter for the sake of clarity. The full periodic buckled beam provides a self-similar load-deformation relationship reducing the analysis to a single period. The long wave approximation also justifies the modeling of the buckled beam by a chain of masses and nonlinear springs (Fig. 1c). Conservation of linear momentum for the $i^{\text {th }}$ particle requires:

$$
\ddot{u}_{i}=\frac{1}{m}\left(P\left\{\Delta U_{0}-u_{i}+u_{i-1}\right\}-P\left\{\Delta U_{0}-u_{i+1}+u_{i}\right\}\right),
$$

where $\left(\ddot{)}\right.$ ) denotes differentiation in time, $u_{i}$ is the displacement of the $i^{\text {th }}$ mass about its equilibrium, and $\Delta U_{0}$ is the initial relative displacement between two consecutive supports. In Eq. (1), the mass $m=m_{\text {Support }}+m_{\text {Beam }}$ accounts for the support mass and the beam 
portion lumped into $m_{\text {Beam }}=\rho A L$. The normalized load-displacement relationship $P\{\Delta U\}$ is given in Fig. 2 for a slender beam and is identical for both support types. While for large buckling level, $P\{\Delta U\}$ depends only on bending deformations (elastica theory) (Fig. 2a), for small buckling level, $P\{\Delta U\}$ is governed also by axial deformations and initial imperfections (extensible-elastica theory) (Fig. 2b) [39]. Note that within the assumption of a slender beam, shear deformations are neglected. Then $P\{\Delta U\}$ can be expressed in terms of a system of elliptical integrals using the extensible-elastica theory [39], but due to the complexity of these equations, this relation is directly computed using FE in the present paper. Assuming $\Delta u=\Delta U-\Delta U_{0}$ small, a Taylor series expansion of $P\{\Delta U\}$ up to the first nonlinear term reads:

$$
P\{\Delta U\}=P\left\{\Delta U_{0}\right\}+P^{\prime}\left\{\Delta U_{0}\right\} \Delta u+\frac{1}{2} P^{\prime \prime}\left\{\Delta U_{0}\right\} \Delta u^{2}+\mathcal{O}\left\{\Delta u^{3}\right\}
$$

where ( $)^{\prime}$ denotes differentiation with respect to $\Delta U$.
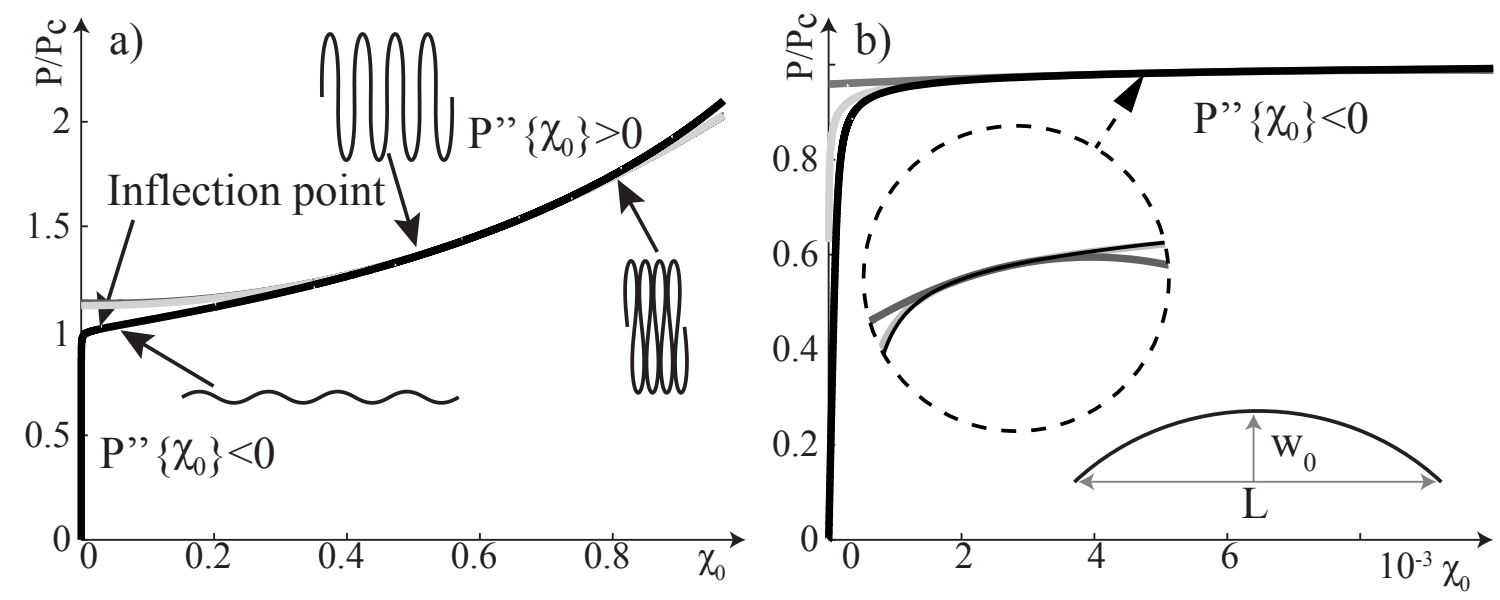

Figure 2: Load-displacement $P\{\Delta U\}$ relationship in black line obtained numerically from FE (see parameters in Sec. 3.2), and normalized by the critical buckling load $P_{c}=\left(n \pi / L_{\text {eff }}\right)^{2} E I_{z}$ where $L_{\text {eff }}=L$ and $L_{\text {eff }}=$ $L / 2$ for respectively pinned-pinned and guided-guided boundary conditions. The figure is plotted in terms of the large (a) and small (b) buckling level $\chi_{0}=\Delta U_{0} / L$. Approximation of the $P\{\Delta U\}$ curve at $\chi_{0}=0.6$ (a) and $\chi_{0}=5 \times 10^{-3}$ (see inset for details) (b) by a polynomial of degree two (Eq. (2)) in dark gray and by a power law in light gray (Eq. (33)).

Plugging Eq. (2) into Eq. (1), homogenizing the discrete momentum equation using finite 
difference and neglecting terms higher than order $\mathcal{O}\left\{L_{0}^{4}\right\}$ lead to [1]:

$$
U_{t t}=C_{0}^{2} U_{x x}+2 C_{0} \gamma U_{x x x x}-\sigma\left(U_{x} U_{x x}\right),
$$

where $C_{0}^{2}=\frac{P^{\prime}\left\{\Delta U_{0}\right\} L_{0}^{2}}{m}$ is the linear speed, $\sigma=\frac{P^{\prime \prime}\left\{\Delta U_{0}\right\} L_{0}^{3}}{m}$ the coefficient of nonlinearity, $\gamma=\frac{C_{0} L_{0}^{2}}{24}$ the coefficient of periodicity-induced dispersion, and $L_{0}=L-\Delta U_{0}$ the length between two consecutive supports after applying pre-compression. Eq. (3) can be recast in terms of the compressive strain $\xi=-U_{x}=-u_{x}+\chi_{0}$, given the initial strain level $\chi_{0}=\Delta U_{0} / L$ also referred as the buckling level:

$$
\xi_{t t}=C_{0}^{2} \xi_{x x}+2 C_{0} \gamma \xi_{x x x x}+\sigma\left(\xi \xi_{x}\right)_{x}
$$

Eq. (4) is the Boussinesq equation [40] and admits the solitary-wave solution called soliton:

$$
\Delta \xi=\Delta \xi_{m} \operatorname{sech}^{2}\left\{\Lambda^{-1}(x-V t)\right\},
$$

where $\Delta \xi=\xi-\chi_{0}, \Delta \xi_{m}=\xi_{m}-\chi_{0}$, and $\xi_{m}$ is the maximum strain. Note that the dynamic wave amplitude $\Delta \xi_{m}$ can be positive (compressive soliton) or negative (tensile or rarefaction soliton) such that $\operatorname{sgn} \Delta \xi_{m}=(\operatorname{sgn} \gamma)(\operatorname{sgn} \sigma)$ and is a very important characteristic that will be revisited in the following sections. The soliton phase speed $V$ is:

$$
V=\sqrt{C_{0}^{2}+\sigma \Delta \xi_{m} / 3}
$$

which is supersonic for $\gamma>0$, the case of pure periodicity dispersion, and is subsonic for $\gamma<0$. The characteristic width $\Lambda$ is

$$
\Lambda=\sqrt{24 C_{0} \gamma /\left(\sigma \Delta \xi_{m}\right)}
$$

and is linked to the width at half maximum $\Lambda_{1 / 2}$ by the relation $\Lambda_{1 / 2}=2 \cosh ^{-1}\{\sqrt{2}\} \Lambda \approx$ 1.7627 . Defining $a_{4}=-\frac{2 \gamma}{C_{0} L_{0}^{2}}$ and $\bar{\sigma}=\frac{\sigma}{2 C_{0}^{2}}$, respectively as the dimensionless dispersive and nonlinear terms, and assuming $a_{4}^{2} \ll 1, \bar{\sigma}^{2} \ll 1$ and $\bar{\sigma} \sim a_{4}$, Eq. (4) can be simplified, following [1], into the KdV equation which also admits the soliton solution Eq. (5) and differs only by the definition of the speed $\left(V=C_{0}+\sigma \Delta \xi_{m} /\left(6 C_{0}\right)\right)$. 
Finally, in [1], we have shown that FE simulations of waves propagating through the structure were in agreement with the Boussinesq equation only for strongly post-buckled beams. For moderately-buckled beams, a front precedes the main waveform as a result of the phase speed of linear waves increasing with frequency, the opposite effect of periodicity dispersion $(\gamma$ in Eq. (4)). Indeed, up to this point, only axial effects have been taken into account, neglecting dispersion sources such as bending effects, displacement coupling, curvature etc., which are discussed in the next section.

\subsection{The semi-analytical dispersion equation}

In order to derive an equation for axial displacements that accounts for all relevant dispersion sources, a FE model is used, first presented in [36]. Here the main steps are revisited to familiarize the reader with the additional coefficients of the dispersion relation. The discretized equations of motion from $\mathrm{FE}$ for one unit cell, given for the nodal displacement vector $\boldsymbol{D}=\boldsymbol{D}_{0}+\boldsymbol{d}$, with $\boldsymbol{D}_{0}$ the displacement at the equilibrium point (buckled configuration) are [41]:

$$
\mathrm{M}\{\boldsymbol{D}\} \ddot{\boldsymbol{D}}+\mathrm{K}\{\boldsymbol{D}\} \boldsymbol{D}=\boldsymbol{F},
$$

where $\mathrm{M}$ and $\mathrm{K}$ are respectively the mass and stiffness matrices of one unit cell, and $\boldsymbol{F}$ is the nodal-force vector. Eq. (8) can be linearized about the equilibrium $\boldsymbol{D}_{0}$, leading to [36]:

$$
\mathrm{M}\left\{\boldsymbol{D}_{0}\right\} \ddot{\boldsymbol{d}}+\mathrm{K}_{T}\left\{\boldsymbol{D}_{0}\right\} \boldsymbol{d}=\boldsymbol{f}
$$

where $\mathrm{K}_{T}\left\{\boldsymbol{D}_{0}\right\}=\mathrm{K}\left\{\boldsymbol{D}_{0}\right\}+\left.\boldsymbol{D}_{\mathbf{0}} \frac{\partial \mathrm{K}\{\boldsymbol{D}\}}{\partial \boldsymbol{D}}\right|_{\boldsymbol{D}_{0}}, \boldsymbol{F}=\mathrm{K}\left\{\boldsymbol{D}_{0}\right\} \boldsymbol{D}_{0}+\boldsymbol{f}$, and $\boldsymbol{d}$ and $\boldsymbol{f}$ are the displacements and forces about equilibrium. Assuming harmonic motion with frequency $\omega$, Eq. (9) gives:

$$
\mathrm{D}\{\omega\} \boldsymbol{d}=\left[\begin{array}{ccc}
\mathrm{D}_{L L} & \mathrm{D}_{L I} & \mathrm{D}_{L R} \\
\mathrm{D}_{I L} & \mathrm{D}_{I I} & \mathrm{D}_{I R} \\
\mathrm{D}_{R L} & \mathrm{D}_{R I} & \mathrm{D}_{R R}
\end{array}\right]\left\{\begin{array}{l}
\boldsymbol{d}_{L} \\
\boldsymbol{d}_{I} \\
\boldsymbol{d}_{R}
\end{array}\right\}=\left\{\begin{array}{c}
\boldsymbol{f}_{L} \\
\boldsymbol{f}_{I} \\
\boldsymbol{f}_{R}
\end{array}\right\}=\boldsymbol{f},
$$

where $\mathrm{D}\{\omega\}=\mathrm{K}_{\mathrm{T}}\left\{\boldsymbol{D}_{0}\right\}-\omega^{2} \mathrm{M}\left\{\boldsymbol{D}_{0}\right\}$ is the dynamic-stiffness matrix. The subscripts ( ) $L$, ()$_{I}$ and ()$_{R}$ respectively denote the left, internal and right displacements/forces of a unit 
cell, and for a sake of clarity, the $T$ of $\mathrm{K}_{T}$ is omitted next. In absence of internal forces, Eq. (10) can be recast as a new reduced dynamic stiffness:

$$
\left[\begin{array}{ll}
\mathcal{D}_{L L} & \mathcal{D}_{L R} \\
\mathcal{D}_{R L} & \mathcal{D}_{R R}
\end{array}\right]\left\{\begin{array}{l}
\boldsymbol{d}_{L} \\
\boldsymbol{d}_{R}
\end{array}\right\}=\left\{\begin{array}{l}
\boldsymbol{f}_{L} \\
\boldsymbol{f}_{R}
\end{array}\right\}
$$

with $(\{X, Y\} \in\{L, R\})$

$$
\mathcal{D}_{X Y}=\mathrm{D}_{X Y}-\mathrm{D}_{X I} \mathrm{D}_{I I}^{-1} \mathrm{D}_{I Y}
$$

Assuming small frequencies $\omega$, the reduced dynamic stiffness can be linearized as

$$
\mathcal{D}_{X Y} \simeq \mathcal{K}_{X Y}-\omega^{2} \mathcal{M}_{X Y}
$$

where the effective stiffness $\mathcal{K}_{X Y}$ and mass $\mathcal{M}_{X Y}$ matrix components are defined by [36]:

$$
\begin{aligned}
& \mathcal{K}_{X Y}=\mathrm{K}_{X Y}-\mathrm{K}_{X I} \mathrm{~K}_{I I}^{-1} \mathrm{~K}_{I Y}, \\
& \mathcal{M}_{X Y}=\mathrm{M}_{X Y}+\mathrm{K}_{X I} \mathrm{~K}_{I I}^{-1} \mathrm{M}_{I I} \mathrm{~K}_{I I}^{-1} \mathrm{~K}_{I Y} .
\end{aligned}
$$

Using Bloch theorem and accounting for glide symmetries [36, 42],

$$
\left\{\begin{array}{c}
\boldsymbol{d}_{L} \\
\boldsymbol{f}_{L}
\end{array}\right\}=e^{-L_{0} \kappa}\left[\begin{array}{cc}
R_{x} & \mathbf{0} \\
\mathbf{0} & R_{x}
\end{array}\right]\left\{\begin{array}{c}
\boldsymbol{d}_{R} \\
-\boldsymbol{f}_{R}
\end{array}\right\}
$$

where $R_{x}$ is defined by:

$$
R_{x}=\left[\begin{array}{ccc}
1 & 0 & 0 \\
0 & -1 & 0 \\
0 & 0 & -1
\end{array}\right]
$$

Combining Eqs. (11) and (15), we have developed in [36] a new technique to obtain a semianalytical dispersion equation for the acoustic branch. This equation is a power series of the frequency $(\omega)$ and the wave number $(\kappa)$ explicitly expressed such that:

$$
\omega^{2}=a_{0,2} C_{0}^{2} \kappa^{2}+L_{0}^{2} C_{0}^{2}\left(a_{0,4}^{(1)} \kappa^{4}+\frac{a_{2,2}^{(1)}}{C_{0}^{2}} \kappa^{2} \omega^{2}+\frac{a_{4,0}^{(1)}}{C_{0}^{4}} \omega^{4}\right),
$$


which is expended up to order four to account for the main dispersive terms. In the case of the guided-support configuration [36],

$$
\begin{aligned}
a_{0,2} & =\frac{1}{\omega_{0}^{2}} \frac{\mathcal{K}_{\text {Tot } 11}}{4 \mathcal{M}_{\text {Tot } 11}}=1, \\
a_{0,4}^{(1)} & =-\frac{1}{12} a_{0,2}=-\frac{1}{12}, \\
a_{2,2}^{(1)} & =\frac{\mathcal{M}_{L R 11}}{2 \mathcal{M}_{\text {Tot } 11}}, \\
a_{4,0}^{(1)} & =0,
\end{aligned}
$$

and for the pinned-supported beam

$$
\begin{aligned}
& a_{0,2}=\frac{1}{\omega_{0}^{2}}\left(\frac{\mathcal{K}_{\text {Tot } 11}}{4 \mathcal{M}_{\text {Tot } 11}}-\frac{\mathcal{K}_{L R 12}^{2}}{\mathcal{M}_{\text {Tot } 11} \mathcal{K}_{\text {Tot } 22}}\right)=1, \\
& a_{0,4}^{(1)}=-\frac{1}{12} a_{0,2}+\frac{\mathcal{K}_{L L 11} \mathcal{K}_{L R 22}+\mathcal{K}_{L R 12}^{2}}{4 \omega_{0}^{2} \mathcal{M}_{\text {Tot } 11} \mathcal{K}_{\text {Tot } 22}}=-\frac{1}{12}+\frac{\mathcal{K}_{L L 11} \mathcal{K}_{L R 22}+\mathcal{K}_{L R 12}^{2}}{\mathcal{K}_{T o t 11} \mathcal{K}_{T o t 22}-4 \mathcal{K}_{L R 12}^{2}}, \\
& a_{2,2}^{(1)}=\frac{\mathcal{M}_{L R 11}}{2 \mathcal{M}_{\text {Tot11 }}}-\frac{\mathcal{K}_{L R 22}}{2 \mathcal{K}_{\text {Tot } 22}}+\frac{8 \mathcal{K}_{L R 12} \mathcal{M}_{L R 12}-\mathcal{K}_{\text {Tot11 }} \mathcal{M}_{\text {Tot } 22}}{4 \mathcal{M}_{\text {Tot11 }} \mathcal{K}_{\text {Tot } 22}}, \\
& a_{4,0}^{(1)}=\frac{\mathcal{M}_{\text {Tot } 22}}{\mathcal{K}_{\text {Tot } 22}} \omega_{0}^{2}=\frac{\mathcal{M}_{\text {Tot } 22}}{\mathcal{M}_{\text {Tot } 11}}\left(\frac{\mathcal{K}_{\text {Tot11 }}}{4 \mathcal{K}_{\text {Tot } 22}}-\frac{\mathcal{K}_{L R 12}^{2}}{\mathcal{K}_{T o t 22}^{2}}\right),
\end{aligned}
$$

such that $\omega_{0}=C_{0} / L_{0}, \mathcal{K}_{T o t 11}=2 \mathcal{K}_{L L 11}, \mathcal{M}_{T o t 11}=\mathcal{M}_{L L 11}+\mathcal{M}_{L R 11}, \mathcal{K}_{T o t 22}=\mathcal{K}_{L L 22}-\mathcal{K}_{L R 22}$ and $\mathcal{M}_{\text {Tot22 }}=\mathcal{M}_{L L 22}-\mathcal{M}_{L R 22}$, where the notation $\mathcal{K}_{X Y i j}$ is the abbreviation for $\mathcal{K}_{X Y}(i, j)$ $(\{i, j\} \in\{1,2\}$ and $\{X Y\} \in\{R L\})$. In the case of the guided-supported beam, in addition to periodicity dispersion $a_{0,4}^{(1)}$ (Fig. 1c), a second term, $a_{2,2}^{(1)}$, is obtained. This term is assigned to transverse inertial effects which can be represented, in the low-frequency regime, by the model shown in Fig. 3a [36]. This means that while phase lags between force and displacement can be neglected, the mass between supports plays an important role even at low frequency. For the case of pinned-supports, in addition to transverse inertia effects, the dispersion relation features additional components accounting for coupling between the support rotation and displacement and its representation is shown in Fig. 3b. It is the goal of the present paper to update traveling-wave solutions in buckled beams including these additional dispersive sources. 

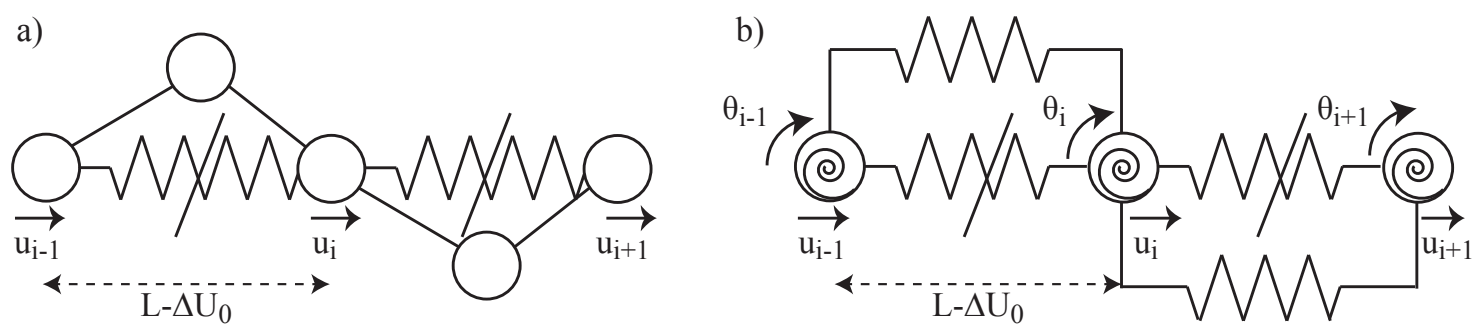

Figure 3: Mechanical models describing (a) transverse inertia effects and (b) support displacements to rotations coupling.

\subsection{Solitary waves in buckled beams including mass and stiffness coupling}

Assuming harmonic waves $\left(\Delta \xi=\Delta \xi_{m} e^{\mathrm{i}(\kappa x-\omega t)}\right)$, a partial differential equation (PDE) is looked for whose dispersion relation corresponds to Eq. (17), and since the equation is linear, the resulting differential equation is:

$$
\xi_{t t}=C_{0}^{2} \xi_{x x}-a_{0,4}^{(1)} L_{0}^{2} C_{0}^{2} \xi_{x x x x}-a_{2,2}^{(1)} L_{0}^{2} \xi_{x x t t}-a_{4,0}^{(1)} \frac{L_{0}^{2}}{C_{0}^{2}} \xi_{t t t t} .
$$

For the guided-support configuration $\left(a_{4,0}^{(1)}=0\right)$, since the supports allows only axial translations, there is no displacement coupling at the support location (see Fig. 3a). In order to include nonlinearity, the nonlinear term from Eq. (4) is taken and added to Eq. (20) describing axial waves such that Eq. (20) becomes the double-dispersion Boussinesq equation:

$$
\xi_{t t}=C_{0}^{2} \xi_{x x}-a_{0,4}^{(1)} L_{0}^{2} C_{0}^{2} \xi_{x x x x}-a_{2,2}^{(1)} L_{0}^{2} \xi_{x x t t}+\sigma\left(\xi \xi_{x}\right)_{x} .
$$

Under the assumption of small amplitude wave and using the regularization technique $\left(\xi_{t t} \approx\right.$ $C_{0}^{2} \xi_{x x}$ ), this equation can be approximated by the simple Boussinesq equation (4), where the dispersive term $\gamma$ is now:

$$
\gamma=-\frac{a_{4} L_{0}^{2} C_{0}}{2} .
$$

with $a_{4}=a_{0,4}^{(1)}+a_{2,2}^{(1)}$ is the dimensionless total dispersion term.

In the case of pinned supports, both axial displacement $U$ and rotation $\theta$ are allowed at the support location (Fig. 3b), so that Eq. (20) is the result of two coupled PDEs. Since the nonlinear term cannot simply be added to Eq. (20) as done for the guided-support case, it 
is the goal here to find the two coupled original PDEs (momentum equations). Combining Eqs. (11) and (15), one gets:

$$
\left[\begin{array}{ll}
D_{11} & D_{12} \\
D_{12} & D_{22}
\end{array}\right]\left\{\begin{array}{l}
U_{m} \\
\theta_{m}
\end{array}\right\}=\mathbf{0}
$$

where $U_{m}$ and $\theta_{m}$ are respectively the amplitude of the harmonic axial strain and rotation waves such that $U=U_{m} e^{i(\kappa x-\omega t)}$ and $\theta=\theta_{m} e^{i(\kappa x-\omega t)}$, and

$$
\begin{aligned}
D_{11} & =\mathcal{D}_{L L 11}+\mathcal{D}_{L R 11} \cosh L_{0} \kappa \\
& =\mathcal{K}_{L L 11}\left(1-\cosh L_{0} \kappa\right)-\mathcal{M}_{\text {Tot } 11} \omega^{2}-\mathcal{M}_{L R 11}\left(\cosh L_{0} \kappa-1\right) \omega^{2}, \\
D_{12} & =\mathrm{i} \mathcal{D}_{L R 12} \sinh L_{0} \kappa \\
& =\mathrm{i}\left(\mathcal{K}_{L R 12}-\mathcal{M}_{L R 12} \omega^{2}\right) \sinh L_{0} \kappa \\
D_{22} & =\mathcal{D}_{L L 22}-\mathcal{D}_{L R 22} \cosh L_{0} \kappa \\
& =\mathcal{K}_{\text {Tot } 22}+\mathcal{K}_{L R 22}\left(1-\cosh L_{0} \kappa\right)-\mathcal{M}_{\text {Tot } 22} \omega^{2}+\mathcal{M}_{L R 22}\left(\cosh L_{0} \kappa-1\right) \omega^{2} .
\end{aligned}
$$

Eq. (23) results from a system of two linear harmonic coupled equations given by:

$$
\begin{aligned}
& \frac{\mathcal{K}_{L L 11}}{2} U_{\bar{x} \bar{x}}-\frac{\mathcal{K}_{L L 11}}{24} U_{\bar{x} \bar{x} \bar{x} \bar{x}}-\mathcal{M}_{T o t 11} U_{t t}+\frac{\mathcal{M}_{L R 11}}{2} U_{\bar{x} \bar{x} t t} \\
& \quad+\mathcal{K}_{L R 12} \theta_{\bar{x}}-\mathcal{M}_{L R 12} \theta_{\bar{x} t t}-\frac{\mathcal{K}_{L R 12}}{6} \theta_{\bar{x} \bar{x} \bar{x}}=0, \\
& \mathcal{K}_{L R 12} U_{\bar{x}}-\frac{\mathcal{K}_{L R 12}}{6} U_{\bar{x} \bar{x} \bar{x}}-\mathcal{M}_{L R 12} U_{\bar{x} t t} \\
& \quad+\mathcal{K}_{T o t 22} \theta-\mathcal{M}_{T o t 22} \theta_{t t}+\frac{\mathcal{K}_{L R 22}}{2} \theta_{\bar{x} \bar{x}}-\frac{\mathcal{K}_{L R 22}}{24} \theta_{\bar{x} \bar{x} \bar{x} \bar{x}}-\frac{\mathcal{M}_{L R 22}}{2} \theta_{\bar{x} \bar{x} t t}=0,
\end{aligned}
$$

where $\bar{x}=x / L_{0}$ is the dimensional space. Eqs. (25) and (26) are respectively the momentum equations describing axial deformations and rotations. The exact solution of this system, up to order four is Eq. (20). However, instead of working with Eq. (20), the axial nonlinear term is added to the axial momentum equation and the new system of coupled PEDs given 
in terms of the strain $\left(\xi=-U_{x}\right)$ and rotation $\theta$ reads:

$$
\begin{aligned}
& \frac{\mathcal{K}_{L L 11}}{2} \xi_{\bar{x} \bar{x}}-\frac{\mathcal{K}_{L L 11}}{24} \xi_{\bar{x} \bar{x} \bar{x} \bar{x}}-\mathcal{M}_{T o t 11} \xi_{t t}+\frac{\mathcal{M}_{L R 11}}{2} \xi_{\bar{x} \bar{x} t t}+\mathcal{M}_{T o t 11} \sigma\left(U^{2}\right)_{x x} \\
& \quad+\frac{1}{L_{0}}\left(-\mathcal{K}_{L R 12} \theta_{\bar{x} \bar{x}}+\mathcal{M}_{L R 12} \theta_{\bar{x} \bar{x} t t}+\frac{\mathcal{K}_{L R 12}}{6} \theta_{\bar{x} \bar{x} \bar{x} \bar{x}}\right)=0 \\
& \mathcal{K}_{L R 12} \xi-\frac{\mathcal{K}_{L R 12}}{6} \xi_{\bar{x} \bar{x}}-\mathcal{M}_{L R 12} \xi_{t t} \\
& \quad+\frac{1}{L_{0}}\left(-\mathcal{K}_{T o t 22} \theta+\mathcal{M}_{T o t 22} \theta_{t t}-\frac{\mathcal{K}_{L R 22}}{2} \theta_{\bar{x} \bar{x}}+\frac{\mathcal{K}_{L R 22}}{24} \theta_{\bar{x} \bar{x} \bar{x} \bar{x}}+\frac{\mathcal{M}_{L R 22}}{2} \theta_{\bar{x} \bar{x} t t}\right)=0 .
\end{aligned}
$$

Using an asymptotic procedure [43, 44], Eq. (28) is recast as:

$$
\frac{\theta}{L_{0}}=\frac{\mathcal{K}_{L R 12}}{\mathcal{K}_{\text {Tot } 22}} \xi-\left(\frac{1}{6} \frac{\mathcal{K}_{L R 12}}{\mathcal{K}_{\text {Tot } 22}}+\frac{1}{2} \frac{\mathcal{K}_{L R 12} \mathcal{K}_{L R 22}}{\mathcal{K}_{\text {Tot } 22}^{2}}\right) \xi_{\bar{x} \bar{x}}-\left(\frac{\mathcal{M}_{L R 12}}{\mathcal{K}_{\text {Tot } 22}}-\frac{\mathcal{K}_{L R 12} \mathcal{M}_{\text {Tot } 22}}{\mathcal{K}_{\text {Tot } 22}^{2}}\right) \xi_{t t} .
$$

Plunging Eq. (29) into Eq. (27), one get:

$$
\xi_{t t}=C_{0}^{2} \xi_{x x}-a_{0,4}^{(2)} L_{0}^{2} C_{0}^{2} \xi_{x x x x}-a_{2,2}^{(2)} L_{0}^{2} \xi_{x x t t}+\sigma\left(\xi \xi_{x}\right)_{x},
$$

where the dimensionless dispersive constants are

$$
\begin{aligned}
& a_{0,4}^{(2)}=-\frac{1}{12}+\frac{\mathcal{K}_{L L 11} \mathcal{K}_{L R 22}+\mathcal{K}_{L R 12}^{2}}{\mathcal{K}_{\text {Tot } 11} \mathcal{K}_{\text {Tot } 22}-4 \mathcal{K}_{L R 12}^{2}}-\frac{\mathcal{K}_{L R 22}}{2 \mathcal{K}_{\text {Tot } 22}} \\
& a_{2,2}^{(2)}=\frac{1}{2} \frac{\mathcal{M}_{L R 11}}{\mathcal{M}_{\text {Tot } 11}}+2 \frac{\mathcal{M}_{L R 12} \mathcal{K}_{L R 12}^{2}}{\mathcal{M}_{\text {Tot } 11} \mathcal{K}_{\text {Tot } 22}}-\frac{\mathcal{M}_{\text {Tot } 22} \mathcal{K}_{L R 12}^{2}}{\mathcal{M}_{\text {Tot } 11} \mathcal{K}_{\text {Tot } 22}^{2}} .
\end{aligned}
$$

Similarly to Eq. (21), the double-dispersion Boussinesq equation (30) can be regularized leading to the simple Boussinesq equation (Eq. (4)) for which $a_{4}=a_{0,4}^{(2)}+a_{2,2}^{(2)}=a_{0,4}^{(1)}+a_{2,2}^{(1)}+a_{4,0}^{(1)}$. For a sake of clarity, the simplified notation is used next; for the guided-support case, $a_{0,4}=a_{0,4}^{(1)}$ and $a_{2,2}=a_{2,2}^{(1)}$, whereas for the pinned-support case, $a_{0,4}=a_{0,4}^{(2)}$ and $a_{2,2}=a_{2,2}^{(2)}$.

In order to compare nonlinearity to dispersion, Eq. (4) is recast into a dimensionless form:

$$
\xi_{\overline{t t}}=\xi_{\bar{x} \bar{x}}+a_{4} \xi_{\bar{x} \bar{x} \bar{x} \bar{x}}+\bar{\sigma}\left(\xi^{2}\right)_{\bar{x} \bar{x}}
$$

with $\bar{\sigma}=\frac{\sigma}{2 C_{0}^{2}}, \bar{x}=\frac{x}{L_{0}}$ and $\bar{t}=\frac{C_{0}}{L_{0}} t$. For both support types, the coefficients of the semianalytical dispersion equation $\left(a_{0,4}, a_{2,2}\right.$, and $\left.a_{4}\right)$ as well as the dimensionless nonlinear term $\bar{\sigma}$ are plotted in Fig. 4 in terms of the pre-compression $\chi_{0}$. 
For the case of guided supports, the first comment due in Fig. 4a is that the main contribution to the total dispersion $\left(a_{4}\right)$ is the transverse inertia $\left(a_{2,2}\right)$ for small buckling level and periodicity $\left(a_{0,4}\right)$ otherwise. The sign of $a_{4}$ is important in that it determines the wave speed behavior in the case of guided supports which is supersonic $\left(a_{4}<0\right)$. For the pinned-support configuration, the main contribution to $a_{4}$ is from $a_{0,4}$ which accounts for both dispersion induced by periodicity and coupling effects. However, since the dimensionless periodicity dispersion is $-1 / 12$ (see Eq. (19)) and is really different from $a_{0,4}$ (Fig. 4b), coupling effects are dominant. Noting now that $a_{4}$ is positive except for extremely large compression, subsonic and supersonic solitary waves are respectively expected for $\chi_{0}>\chi_{\gamma}$ and $\chi_{0}<\chi_{\gamma}$ (see Table 1$)$, where $\chi_{\gamma} \approx 0.85$.

Concerning nonlinearity, its sign also influences the type of solitary wave since sgn $\Delta \xi_{m}=$ $-\left(\operatorname{sgn} a_{4}\right)(\operatorname{sgn} \bar{\sigma})$, where $\bar{\sigma}$ is found negative for $\chi_{0}<\chi_{\sigma}$ and positive for $\chi_{0}>\chi_{\sigma}$ whatever the support configuration (see Fig. 4), and $\chi_{\sigma} \approx 0.081$ is the level of pre-compression for which there is an inflection in the load-displacement curve (Fig. 2).

Combining the sign of dispersive and nonlinear terms, four kinds of waves are possible: compressive supersonic, compressive subsonic, rarefaction supersonic, and rarefaction subsonic waves. What is particularly interesting with buckled beams is that varying the level of the pre-compression and the support type, a configuration for each of the four different wave types can be obtained, as shown in Table 1. Furthermore, with pinned supports, simply varying pre-compression gives three different wave types.

Table 1: Solitary waves characteristics in terms of the sign of the nonlinear $\bar{\sigma}$ and dispersive $a_{4}$ terms (Fig. 4).

\begin{tabular}{|c|c|c|c|c|c|}
\hline $\operatorname{sgn} \bar{\sigma}$ & $\operatorname{sgn} a_{4}$ & Shape behavior & Speed behavior & Configurations & Symbol \\
\hline+ & - & Compression & Supersonic & $\begin{array}{c}\text { Guided: } \chi_{0}>\chi_{\sigma} \\
\text { Pinned: } \chi_{0}>\chi_{\gamma}\end{array}$ & $\nearrow$ \\
\hline- & - & Rarefaction & Supersonic & Guided: $\chi_{0}<\chi_{\sigma}$ & $\searrow$ \\
\hline+ & + & Rarefaction & Subsonic & Pinned: $\chi_{\sigma}<\chi_{0}<\chi_{\gamma}$ & $\swarrow$ \\
\hline- & + & Compression & Subsonic & Pinned: $\chi_{0}<\chi_{\sigma}$ & $\nwarrow$ \\
\hline
\end{tabular}

However, the amplitude range $\Delta \xi_{m}$ for which solitary waves are expected is limited by two phenomena. Indeed, the dynamic wave cannot cross the snapping point $\left(\chi_{0}=0\right)$ or the 


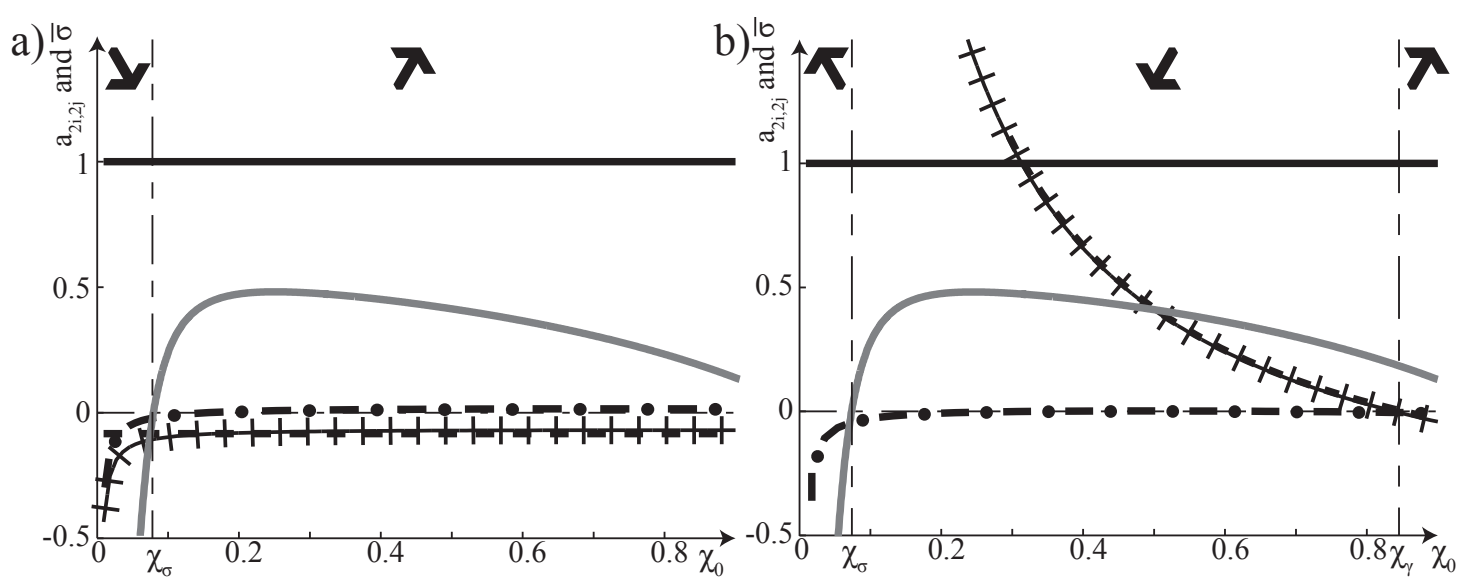

Figure 4: Dimensionless nonlinear and dispersive coefficients, in terms of the pre-compressive strain $\chi_{0}$ for the guided (a) and pinned (b) support configurations. $a_{0,2}=1$ in full, $a_{0,4}$ in dashed, $a_{2,2}$ in dashed-dotted, $a_{4}$ in + dark, and $\bar{\sigma}$ in full gray lines. See Table 1 for arrow notation.

inflection point $\left(\chi_{0}=\chi_{\sigma}\right)$ either in compression or in tension, since the static curve $P\left\{\Delta U_{0}\right\}$ is not defined for $\chi_{0}<0$ and is linear for $\chi_{0}=\chi_{\sigma}$. The second constraint applies only for $a_{4}$ positive (pinned-support configuration with $\chi_{0}<\chi_{\gamma}$ ) and is such that the subsonic wave speed has to remain real (see Eq. (6)). While the equations governing the constraints on $\Delta \xi_{m}$ are given in Table 2, numerical results are shown in Fig. 5.

Table 2: Constraints on the upper bound of the wave amplitude $\left|\Delta \xi_{m}\right|$ given by the fact that the dynamic wave cannot cross the snapping point $\left(\chi_{0}=0\right)$ or the inflection point $\left(\chi_{0}=\chi_{\sigma}\right)$ and also limited by the fact that the subsonic speed cannot be imaginary.

\begin{tabular}{|c|c|c|}
\hline Configuration & $P\left\{\Delta U_{0}\right\}$ & Speed real (Eq. (6)) \\
\hline Guided: $\chi_{0}<\chi_{\sigma}$ & $\left|\Delta \xi_{m}\right|<\chi_{0} L / L_{0}$ & $\varnothing$ \\
\hline Guided: $\chi_{0}>\chi_{\sigma}$ & $\varnothing$ & $\varnothing$ \\
\hline Pinned: $\chi_{0}<\chi_{\sigma}$ & $\left|\Delta \xi_{m}\right|<\left(\chi_{\sigma}-\chi_{0}\right) L / L_{0}$ & $\left|\Delta \xi_{m}\right|<3 C_{0}^{2} / \sigma$ \\
\hline Pinned: $\chi_{\sigma}<\chi_{0}<\chi_{\gamma}$ & $\left|\Delta \xi_{m}\right|<\left(\chi_{0}-\chi_{\sigma}\right) L / L_{0}$ & $\left|\Delta \xi_{m}\right|<3 C_{0}^{2} / \sigma$ \\
\hline Pinned: $\chi_{0}>\chi_{\gamma}$ & $\varnothing$ & $\varnothing$ \\
\hline
\end{tabular}

To conclude, it is shown here that updating dispersion by including mass and stiffness coupling leads again to a solitary wave. The variety of wave types in buckled-beams is evident in that four different waves (rarefaction/compression shapes and supersonic/subsonic speeds) can be obtained changing only the support-type and the pre-compression level (Table 1). The goal is next to investigate nonlinear wave propagation for large amplitude or small 

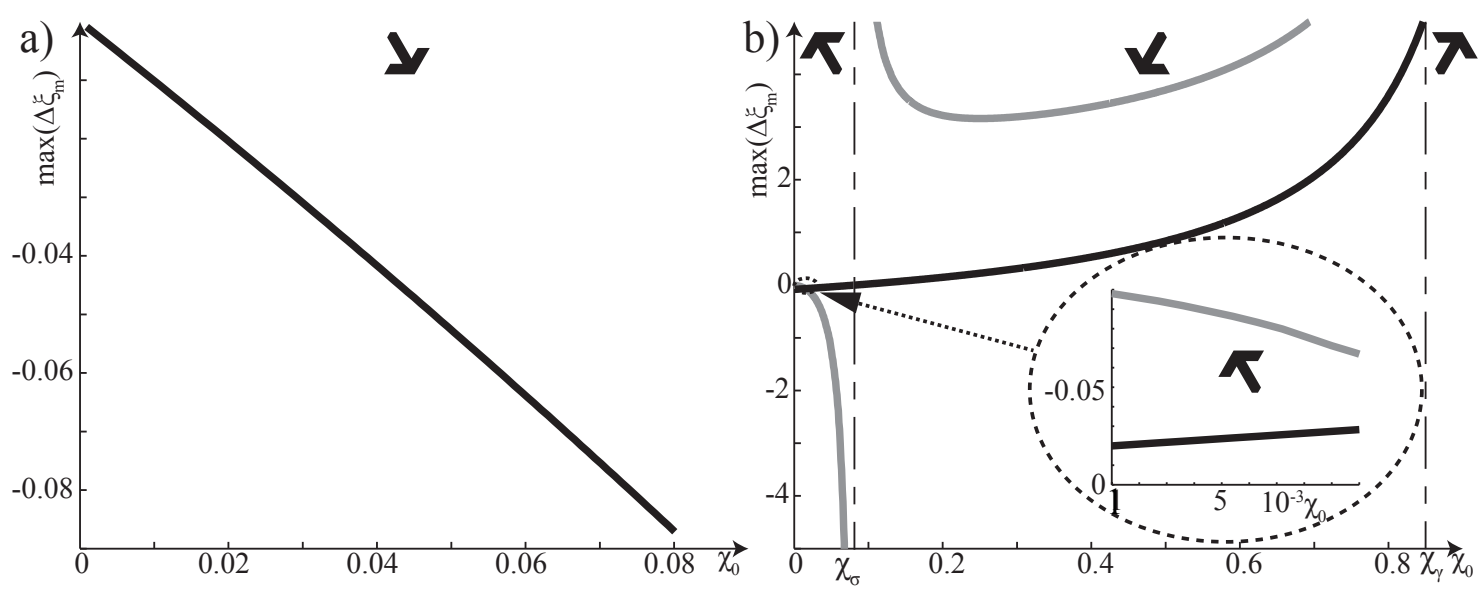

Figure 5: Maximum value of $\Delta \xi_{m}$ for the guided (a) and pinned-support (b) configurations in terms of the pre-compression $\chi_{0}$ computed using the rules given in Table 2. Limitations arising from the load-deformation behavior shown in black lines, and real speed in gray lines. See Table 1 for arrow notation.

pre-compression, where the Boussinesq model is not valid.

\subsection{Strongly nonlinear wave propagation}

For nonlinear waves with large amplitude or small pre-compression, the approximation of the load-displacement $P\{\Delta U\}$ by a second degree polynomial (Eq. (2)) is not accurate (see Fig. 2) but is used in the derivation of the Boussinesq model. In this section, the approximation of $P\{\Delta U\}$ by a power law is proposed. Where a power law or polynomial are not sufficient, $P\{\Delta U\}$ is retained as a general function.

As a general comment, for no pre-stress, the load-displacement relation is governed by axial deformations, such that its behavior is linear (see Fig. 2). Its means that the structure will not act as a "sonic vacuum" [11], and it is the reason why pre-compression is always considered here.

\subsubsection{Nonlinearity described by power law}

Wave propagation in nonlinear media where the nonlinearity can be described locally by a power law

$$
P\{\Delta U\}=a \Delta U^{n}+b,
$$


is quite common and arises for example in describing wave propagation in granular media $[11,12]$ with $n=3 / 2$ for spheres (Hertz law), and any $n>0$ when the contact surfaces are not elliptical (Hertz-like law [45]). Here, the coefficients of Eq. (33) are given in terms of the local derivatives of the load-displacement such that $a=\frac{1}{m} \frac{P^{\prime}\left\{\Delta U_{0}\right\}}{n U_{0}^{n-1}}, n=1+\Delta U_{0} \frac{P^{\prime \prime}\left\{\Delta U_{0}\right\}}{P^{\prime}\left\{\Delta U_{0}\right\}}$ and $b=P\left\{\Delta U_{0}\right\}-a \Delta U_{0}^{n}$, and note that as in Eq. (2), these coefficients refer up to the second derivative of $P\{\Delta U\}$. Note also that for $\chi_{0}<\chi_{n} \approx 8.8 \times 10^{-3}, n$ and $a$ are both negative, as shown in Fig. 6.

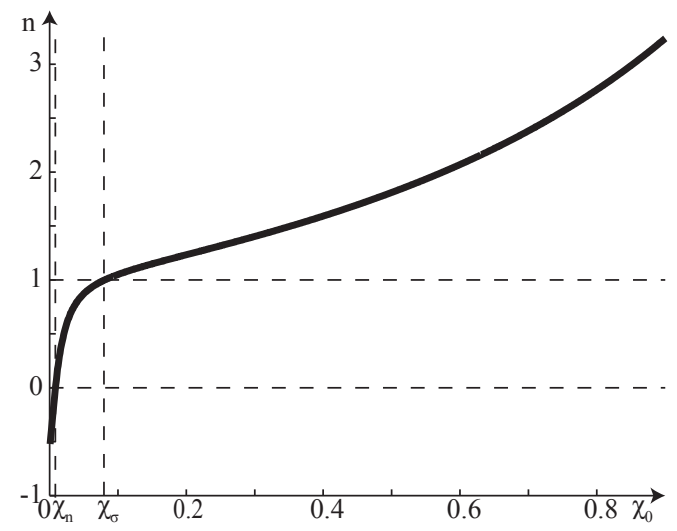

Figure 6: Power $n$ of Eq. (33) in terms of the pre-compression $\chi_{0}$. Note that $n>1$ for $\chi_{0}>\chi_{\sigma}, n<0$ for $\chi_{0}<\chi_{n}$ and $n \in\left[\begin{array}{ll}0 & 1\end{array}\right]$ otherwise.

Nesterenko [11] shows that chains of masses and nonlinear springs (Fig. 1c, and Eq. (1)) with the load-displacement $P\{\Delta U\}$ given by Eq. (33) are capable of hosting stable solitary waves. In this paper, this work will be extended to account for the additional dispersion sources. For the case of a buckled beam on guided supports $\left(a_{0,4}=-1 / 12\right)$ modeled by a simple one-dimensional chain of nonlinear springs and masses, with the nonlinearity described by a power law, the momentum equation governing the $i^{\text {th }}$ particle is:

$$
\ddot{u}_{i}=\frac{a}{m}\left(\left(U_{i-1}-U_{i}\right)^{n}-\left(U_{i}-U_{i+1}\right)^{n}\right) .
$$

In the long wave approximation, Eq. (34) can be homogenized into a strongly nonlinear wave equation [11]:

$$
\xi_{t t}=C_{n}^{2}\left(\xi^{n}-a_{0,4} L_{0}^{2} \frac{2 n}{n+1} \xi^{\frac{n-1}{2}}\left(\xi^{\frac{n+1}{2}}\right)_{x x}\right)_{x x}-L_{0}^{2} a_{2,2} \xi_{x x t t},
$$


where $C_{n}^{2}=\frac{a}{m} L_{0}^{n+1}$ and $\xi=-U_{x}$. While the first part of the right hand side accounts for nonlinearity and periodicity dispersion, the last term is added a posteriori to account for dispersion due to transverse inertial effects. Using the regularization $\xi_{t t} \approx C_{n}^{2}\left(\xi^{n}\right)_{x x}$, Eq. (35) can be recast in:

$$
\xi_{t t}=C_{n}^{2}\left(\xi^{n}-a_{0,4} L_{0}^{2} \frac{2 n}{n+1} \xi^{\frac{n-1}{2}}\left(\xi^{\frac{n+1}{2}}\right)_{x x}-a_{2,2} L_{0}^{2}\left(\xi^{n}\right)_{x x}\right)_{x x} .
$$

Using the transformation

$$
\left(\xi^{n}\right)_{x x}=\frac{2 n}{n+1} \xi^{\frac{n-1}{2}}\left(\xi^{\frac{n+1}{2}}\right)_{x x}+\frac{n(n-1)}{2} \xi^{n-2} \xi_{x}^{2},
$$

Eq. (36) can be rewritten into two different forms:

$$
\begin{aligned}
\xi_{t t} & =C_{n}^{2}\left(\xi^{n}-a_{4} L_{0}^{2} \frac{2 n}{n+1} \xi^{\frac{n-1}{2}}\left(\xi^{\frac{n+1}{2}}\right)_{x x}-a_{2,2} L_{0}^{2} \frac{n(n-1)}{2} \xi^{n-2} \xi_{x}^{2}\right)_{x x} \\
& =C_{n}^{2}\left(\xi^{n}-a_{4} L_{0}^{2}\left(\xi^{n}\right)_{x x}+a_{0,4} L_{0}^{2} \frac{n(n-1)}{2} \xi^{n-2} \xi_{x}^{2}\right)_{x x} .
\end{aligned}
$$

However, contrary to [11], due to the additional term in $a_{2,2}$, Eqs. (38) or (39) cannot be recast exactly into potential form, and it is necessary to neglect the last terms of these equations. Justification of this assumption will be given a posteriori by showing that results from truncated forms of Eqs. (38) and (39) are similar. In addition, for $\left|a_{4}\right| \gg\left|a_{2,2}\right|$, neglecting $a_{2,2}$ in Eq. (39) leads to Eq. (38) without its last term. Similarly, the use of the assumption $\left|a_{4}\right| \gg\left|a_{0,4}\right|$ in Eq. (38) leads to the truncated form of Eq. (39). Since the ratio $\left|a_{2,2} / a_{0,4}\right|$ is large for weakly buckled beams and small otherwise (Fig. 4), both equations are considered next.

Potential for Eq. (38)

Neglecting the last term of Eq. (38), this equation becomes identical to the one studied in [11], and for $n>1$ and $0<n<1$, respectively compressive and rarefaction solitary waves are possible solutions. However, for $n<0$, since $a<0, C_{n}=\sqrt{\frac{a}{m} L_{0}^{n+1}}$ is purely imaginary. Note that the long wave sound speed $C_{0}=j C_{n} \sqrt{n} \xi_{0}^{\frac{n-1}{2}}$ with $j=\operatorname{sgn} n$, remains 
real $\left(\xi_{0}=\Delta U_{0} / L_{0} \neq \chi_{0}=\Delta U_{0} / L\right)$. In order to recast Eq. (38) in form of a potential $W\{z\}$ such that

$$
z_{x x}=-\frac{d W\{z\}}{d z}
$$

Nesterenko uses the change of variables $z=\xi^{\frac{n+1}{2}}\left(\frac{C_{n}}{V}\right)^{\frac{n+1}{n-1}}$ but with $n<0, z$ becomes a complex number and the one-to-one relation between $z$ and $\xi$ is lost. In the present paper, an update in the change of variables is proposed and reads:

$$
z=\xi^{\frac{n+1}{2}}\left(j\left(\frac{C_{n}}{V}\right)^{2}\right)^{\frac{n+1}{2(n-1)}} .
$$

In the case of stationary waves, $z=z\{x-V t\}$ and using the boundary conditions

$$
\lim _{x= \pm \infty} \xi=\xi_{0} \text { and } \lim _{x= \pm \infty} \xi_{x}=\lim _{x= \pm \infty} \xi_{x x}=0
$$

Eq. (38) can be rewritten in the form of a potential $W\{z\}$ :

$$
W\{z\}=-\frac{n+1}{2 n a_{4} L_{0}^{2}}\left(\frac{1}{2} z^{2}-j \frac{n+1}{4} z^{\frac{4}{n+1}}+C z^{\frac{2}{n+1}}\right) .
$$

Using the fact that $\left.z_{x x}\right|_{\xi=\xi_{0}}=0$, the constant $C$ is

$$
C=\frac{n+1}{2}\left(j z_{0}^{\frac{2}{n+1}}-z_{0}^{\frac{2 n}{n+1}}\right)
$$

where $z_{0}=z\left\{\xi_{0}\right\}$. In order to have localized stationary waves, the potential must have two extremes and following the procedure in [11], $C$ has to comply with Table 3 which is validated numerically for the full range of compression levels $\left(0<\chi_{0}<0.9\right)$. The shape of

Table 3: Constraints on $C$ derived following [11] and with $N_{1}=\frac{n^{2}-1}{2}(j n)^{n /(1-n)}$.

\begin{tabular}{|c|c|c|c|}
\hline $1<n$ & $0<n<1$ & $-1<n<0$ & $n<-1$ \\
\hline $0<C<N_{1}$ & $N_{1}<C<0$ & $C<N_{1}$ & $N_{1}<C$ \\
\hline
\end{tabular}

the solitary wave is obtained by recasting Eq. (40) into an elliptical integral

$$
x=\int_{z}^{z_{m}} \frac{d z}{\sqrt{2\left(W\left\{z_{0}\right\}-W\{z\}\right)}},
$$


which is solved numerically and $z_{m}=z\left\{\xi_{m}\right\}$ where $\xi_{m}$ is the maximum strain. In order to compare the wavelength of this solitary wave to simulations, the width of the wave at half of its amplitude is used and reads:

$$
\Lambda_{1 / 2}=2 \int_{\frac{z_{0}+z_{m}}{2}}^{z_{m}} \frac{d z}{\sqrt{2\left(W\left\{z_{0}\right\}-W\{z\}\right)}} .
$$

Using the fact that $W\left\{\xi_{m}\right\}=W\left\{\xi_{0}\right\}$, the solitary wave speed is:

$$
V=\frac{C_{n}}{\left|\xi_{m}-\xi_{0}\right|}\left(2 \frac{n \xi_{0}^{n+1}+\xi_{m}^{n+1}-(n+1) \xi_{0}^{n} \xi_{m}}{n+1}\right)^{1 / 2} .
$$

Note that this equation can be directly recovered from [11] using a general nonlinear function. recovered

Potential for Eq. (39)

The same procedure developed in Sec. 2.4.1 can be applied to Eq. (39) using the change of variable:

$$
z=\xi^{n}\left(j\left(\frac{C_{n}}{V}\right)^{2}\right)^{\frac{n}{n-1}} .
$$

Eq. (39) can be rewritten in potential form:

$$
W\{z\}=-\frac{1}{a_{4} L_{0}^{2}}\left(\frac{1}{2} z^{2}-j \frac{n}{n+1} z^{\frac{n+1}{n}}+C z\right),
$$

where the constant $C=j z_{0}^{1 / n}-z_{0}$ has to fulfill the conditions given in Table 4 which are validated numerically for the compression level $0<\chi_{0}<0.9$. The shape of the solitary wave

Table 4: Constraints on $C$ with $N_{2}=(n-1)(j n)^{n /(1-n)}$.

\begin{tabular}{|c|c|c|}
\hline $1<n$ & $0<n<1$ & $n<0$ \\
\hline $0<C<N_{2}$ & $C<N_{2}<0$ & $C<N_{2}$ \\
\hline
\end{tabular}

is given by Eq. (45) and the solitary wave speed is:

$$
V=C_{n}\left|\xi_{m}^{n}-\xi_{0}^{n}\right|\left(\frac{n+1}{2\left(\xi_{0}^{n+1}+n \xi_{m}^{n+1}-(n+1) \xi_{0} \xi_{m}^{n}\right)}\right)^{1 / 2} .
$$


For the sake of clarity, up to this point, only the case of guided supports has been investigated (see Eq. (34)). Pinned-support configurations can be considered as well but the $z=z\{\xi\}$ relation is not invertible analytically anymore. As a consequence, it is not possible to get analytical expressions for the potential or the phase speed, so that the simplicity of using the power-law approximation compared to the use of the general relation $P\{\Delta U\}$ is lost, as it will be shown in next section.

\subsubsection{Nonlinearity described by general function}

Instead of approximating the load-displacement relationship by an analytical equation (second degree polynomial Eq. (2) or power-law Eq. (33)), in this section this relation is kept general. The momentum equation of the $i^{\text {th }}$ particle of the one-dimensional chain is:

$$
\ddot{u}_{i}=\frac{1}{m}\left(P\left\{U_{i-1}-U_{i}\right\}-P\left\{U_{i}-U_{i+1}\right\}\right) .
$$

This equation, in the case of dispersion induced purely by periodicity $\left(a_{0,4}^{\mathrm{No} C_{0}}=a_{2,2}=0\right)$, has been already analyzed in [11] and following the same procedure, Eq. (51) is homogenized into a nonlinear wave equation:

$$
\xi_{t t}=\frac{1}{\rho_{0}}\left(P-a_{0,4}^{C_{0}} L_{0}^{2} \sqrt{P^{\prime}}\left(\int_{\xi_{0}}^{\xi}\left(P^{\prime}\right)^{1 / 2} d \xi\right)_{x x}\right)_{x x}-L_{0}^{2}\left(a_{0,4}^{\mathrm{No} C_{0}} C_{0}^{2} \xi_{x x x x}+a_{2,2} \xi_{x x t t}\right),
$$

where $\rho_{0}=\frac{m}{L_{0}}, P=P\left\{L_{0} \xi\right\}$ and ( )' denotes now the derivative with respect to $\xi$. While the first part of the right hand side accounts for nonlinearity and dispersion proportional to the linear speed, the following two terms added a posteriori account for the rest of the dispersion sources with $a_{0,4}=a_{0,4}^{C_{0}}+a_{0,4}^{\mathrm{No} C_{0}}$, and $a_{0,4}^{C_{0}}=-\frac{1}{12} \cdot a_{0,4}^{\mathrm{No} C_{0}}$ is null for the case of the guided supports and defined by Eq. (19) for the case of the pinned supports. Using the equality $C_{0}^{2}=\frac{P_{0}^{\prime}}{\rho_{0}}$ where $P_{0}=\left.P\left\{L_{0} \xi\right\}\right|_{\xi=\xi_{0}}$, the regularization method $\left(\xi_{t t} \approx P_{x x} / \rho_{0}\right)$ leads to:

$$
\xi_{t t}=\frac{L_{0}^{2}}{\rho_{0}}\left(\frac{P}{L_{0}^{2}}-a_{0,4}^{C_{0}} \sqrt{P^{\prime}}\left(\int_{\xi_{0}}^{\xi}\left(P^{\prime}\right)^{1 / 2} d \xi\right)_{x x}-a_{2,2} P_{x x}-a_{0,4}^{\mathrm{No} C_{0}} P_{0}^{\prime} \xi_{x x}\right)_{x x} .
$$


Note that using the change of variable $\xi=\xi_{0}+\Delta \xi$, expansion of Eq. (36) into series assuming $\Delta \xi$ small gives back the Boussinesq equation (Eq. (4)). Using the transformations

$$
P_{x x}=\sqrt{P^{\prime}}\left(\int_{\xi_{0}}^{\xi}\left(P^{\prime}\right)^{1 / 2} d \xi\right)_{x x}+\frac{P^{\prime \prime}}{2} \xi_{x}^{2},
$$

and

$$
\xi_{x x}=\sqrt{P^{\prime}}\left(\int_{\xi_{0}}^{\xi}\left(P^{\prime}\right)^{-1 / 2} d \xi\right)_{x x}+\frac{P^{\prime \prime}}{2 P^{\prime}} \xi_{x}^{2},
$$

Eq. (53) can be recast into two different forms:

$$
\begin{aligned}
\xi_{t t}= & \frac{L_{0}^{2}}{\rho_{0}}\left(\frac{P}{L_{0}^{2}}-\sqrt{P^{\prime}}\left(\left(a_{2,2}+a_{0,4}^{C_{0}}\right) \int_{\xi_{0}}^{\xi}\left(P^{\prime}\right)^{1 / 2} d \xi+a_{0,4}^{\mathrm{No} C_{0}} P_{0}^{\prime} \int_{\xi_{0}}^{\xi}\left(P^{\prime}\right)^{-1 / 2} d \xi\right)_{x x}\right. \\
& \left.-\xi_{x}^{2}\left(a_{2,2} \frac{P^{\prime \prime}}{2}+a_{0,4}^{\mathrm{No} C_{0}} \frac{P_{0}^{\prime} P^{\prime \prime}}{2 P^{\prime}}\right)\right)_{x x} \\
= & \frac{L_{0}^{2}}{\rho_{0}}\left(\frac{P}{L_{0}^{2}}-\left(\left(a_{2,2}+a_{0,4}^{C_{0}}\right) P+a_{0,4}^{\mathrm{NoC} C_{0}} P_{0}^{\prime} \xi\right)_{x x}+a_{0,4} \frac{P^{\prime \prime}}{2} \xi_{x}^{2}\right)_{x x} .
\end{aligned}
$$

In order to recast these equations into potential form, the last term of Eqs. (56) or (57) has to be neglected, as done in Sec. 2.4.1 and is justified a posteriori.

Using the variable transformation $z=\left(a_{2,2}+a_{0,4}^{C_{0}}\right) \int_{\xi_{0}}^{\xi}\left(P^{\prime}\right)^{1 / 2} d \xi+a_{0,4}^{\mathrm{No} C_{0}} P_{0}^{\prime} \int_{\xi_{0}}^{\xi}\left(P^{\prime}\right)^{-1 / 2} d \xi$ for Eq. (56) or $z=\left(a_{2,2}+a_{0,4}^{C_{0}}\right) P+a_{0,4}^{\mathrm{NoC} C_{0}} P_{0}^{\prime} \xi$ for Eq. (57), in the case of stationary waves $z=z\{x-V t\}$, and using the boundary conditions of Eq. (42),

$$
\frac{d W\{z\}}{d z}=-\frac{1}{L_{0}^{2} F}\left(V^{2} \rho_{0}\left(\xi-\xi_{0}\right)-\left(P-P_{0}\right)\right),
$$

where $\xi=\xi\{z\}$ and $F=\sqrt{P^{\prime}}$ or $F=1$, respectively for Eq. (56) and Eq. (57). The speed is derived using the equality $W\left\{\xi_{m}\right\}=W\left\{\xi_{0}\right\}$ and reads:

$$
V^{2}=\frac{1}{\rho_{0}} \frac{\int_{z_{0}}^{z_{m}} \frac{P-P_{0}}{F} d z}{\int_{z_{0}} \frac{\xi-\xi_{0}}{F} d z},
$$

where $z_{0}=z\left\{\xi_{0}\right\}, z_{m}=z\left\{\xi_{m}\right\}$, and the shape of the solitary wave is obtained using Eq. (45). Note that contrary to the case of the power-law nonlinearity (Sec. 2.4.1), even for the guidedsupports case $\left(a_{0,4}^{\mathrm{NoC}}=0\right)$, the relation $z=z\{\xi\}$ is not invertible analytically meaning that 
the expression of the speed can be obtained only using numerical integration (Eq. (59)). The validity of these different models is investigated using FE simulations, which are introduced next.

\section{Finite-Element Models and Parameters}

\subsection{Finite-element models}

The selected beam formulation is the co-rotational model [46] which is limited to small strain (linear elastic material) but applicable to finite displacements and rotations. Since the beam is assumed slender, this formulation is used neglecting shear. A super-convergent beam formulation is discussed in [47], but given the need to use Lagrange multipliers to enforce boundary conditions, a co-rotational formulation is employed here for simplicity. For dynamic problems, the generalized- $\alpha$ method is employed as the time integration scheme, which can be second-order accurate in time and unconditionally stable for linear problems [48] (see [49] for details of the method and choice of parameters). Specifically, the parameters

used for the method are considered dependent on $\rho_{\infty} \in\left[\begin{array}{ll}0 & 1\end{array}\right]$, which is the high-frequency dissipation parameter. Nonlinear equilibrium states are obtained using the Newton-Raphson method [41].

\subsection{Geometric and material properties}

The values of the different parameters correspond to the physical parameters used in experiments discussed in [38]. The beam has a rectangular cross section of area $A=b t$ and area-moment of inertia $I_{z}=b t^{3} / 12$ where $t=0.4 \mathrm{~mm}$ and $b=12.7 \mathrm{~mm}$. The material is steel assumed to be linear and elastic with $E=190 \mathrm{MPa}$ and $\rho=7600 \mathrm{Kg} \mathrm{m}^{-3}$. The length between each support is initially $L=60 \mathrm{~mm}$ and the mass of one support is $m_{\text {Support }}=24$ g. The initial imperfection is chosen as $w_{0}=10^{-3}$ (see Fig. 2b).

For numerical simulations, each wavelength is composed of 30 or 8 beam elements and a total of $p=300$ or $p=1200$ wavelengths are used for respectively the guided and pinnedsupport configurations. Simulations are divided into two steps; the first one in load con- 
trol where the structure is statically buckled after applying the desired load for the precompression, and a second one, in displacement control, where a dynamic pulse is generated at the left boundary. Both static and dynamic simulations are divided into 5000 steps (in the static case this is done to ensure resolution in the $P\{U\}$ curve) and the convergence criterion for the Newton-Raphson scheme is defined by $\left\|R_{i}\right\|_{\infty} /\left\|R_{0}\right\|_{\infty}<10^{-5}$ or $\left\|R_{i}\right\|_{\infty}<10^{-10}$ where $R_{0}$ is the residual due to the external forces and $R_{i}$ is the residual at the convergence iteration $i$. In dynamics, the high-frequency dissipation parameter is chosen as $\rho_{\infty}=0.9$ allowing convergence with limited numerical dissipation.

Numerical simulations are performed imposing the dynamic displacement on the left extremity of the beam $U_{\text {Left }}$ (Fig. 1), obtained after integrating Eq. (5) in space such that:

$$
U_{\text {Left }}=\Delta \xi_{m} \Lambda\left(\tanh \left\{\Lambda^{-1} V\left(t_{0}-t\right)\right\}+1\right)+p \Delta U_{0},
$$

where $t_{0}$ is chosen arbitrarily as $t_{0}=5 \Lambda / V$. The total time of the dynamic simulation is given by $t_{f}=p L / V$ where $V$ is computed from Eq. (4).

\section{Results And Discussion}

\subsection{Comparison of the Boussinesq models to FE simulations \\ 4.1.1. Guided-supported beam}

Snapshots of the strain wave propagating through the guided-support beam for several levels of compression are shown in Fig. 7 where the input is either a compressive or a rarefaction wave, depending on the compression level (Table 1). In Figs. 7c,f, the wave propagates without any change in shape, in agreement with the stationary properties of solitary waves. In Figs. 7a-d, a small tail is present and propagates more slowly than the main wave, in agreement with the supersonic properties predicted by the Boussinesq model (Table 1). If the input does not lead directly to the asymptotic solution then this input simply does not correspond to the stationary solution of the Boussinesq model. This reason is particularly probable in Fig. 7d where the initial guess splits into two distinct waves.

Additionally, in order to check if stationary waves are indeed nonlinear, the dependency of speed and wavelength on amplitude is shown in Fig. 8 in terms of the compression $\chi_{0}$ and 

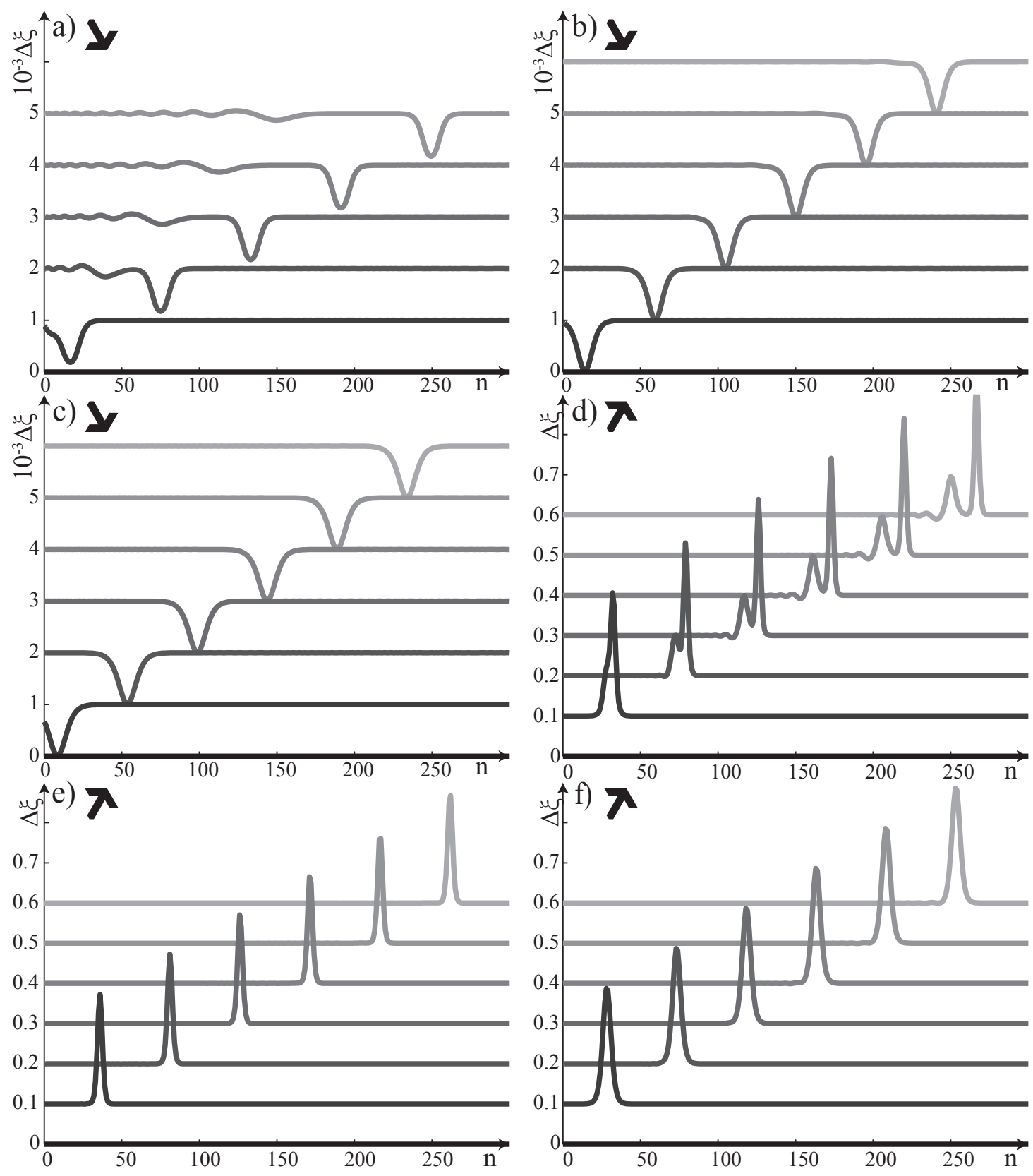

Figure 7: Snapshots at times multiple of $t_{i}=3 / 20 t_{f}$ of the strain wave propagating in the guided-supported buckled beam with the pre-compression $\chi_{0}=1 \times 10^{-3}$ (a), $\chi_{0}=5 \times 10^{-3}$ (b), $\chi_{0}=10 \times 10^{-3}$ (c), $\chi_{0}=0.1$ (d), $\chi_{0}=0.5(\mathrm{e})$ and $\chi_{0}=0.9$ (f), with dynamic input rarefaction $\Delta \xi_{m}=-1 \times 10^{-3}$ (a,b,c) and compressive $\Delta \xi_{m}=0.3$ (e,f,g) waves. See Table 1 for arrow notation.

the dynamic-amplitude $\Delta \xi_{m}$. Figs. 8a,b show that the speed indeed increases whereas the wavelength decreases with the absolute amplitude, in agreement with the Boussinesq model. 
However, for weakly-compressed beams or large wave amplitudes, the values of the speed and wavelength extracted from FE simulations are not in agreement with the Boussinesq equation (Eq. (4)). This is due to the fact that for certain conditions, approximation of the load-displacement curve by a polynomial of degree two (Eq. (2)) is not accurate. Focusing now on the wavelength, it is found that the characteristic width decreases when the dynamic wave amplitude increases and that the FE results match the Boussinesq model (Figs. 8c,d).
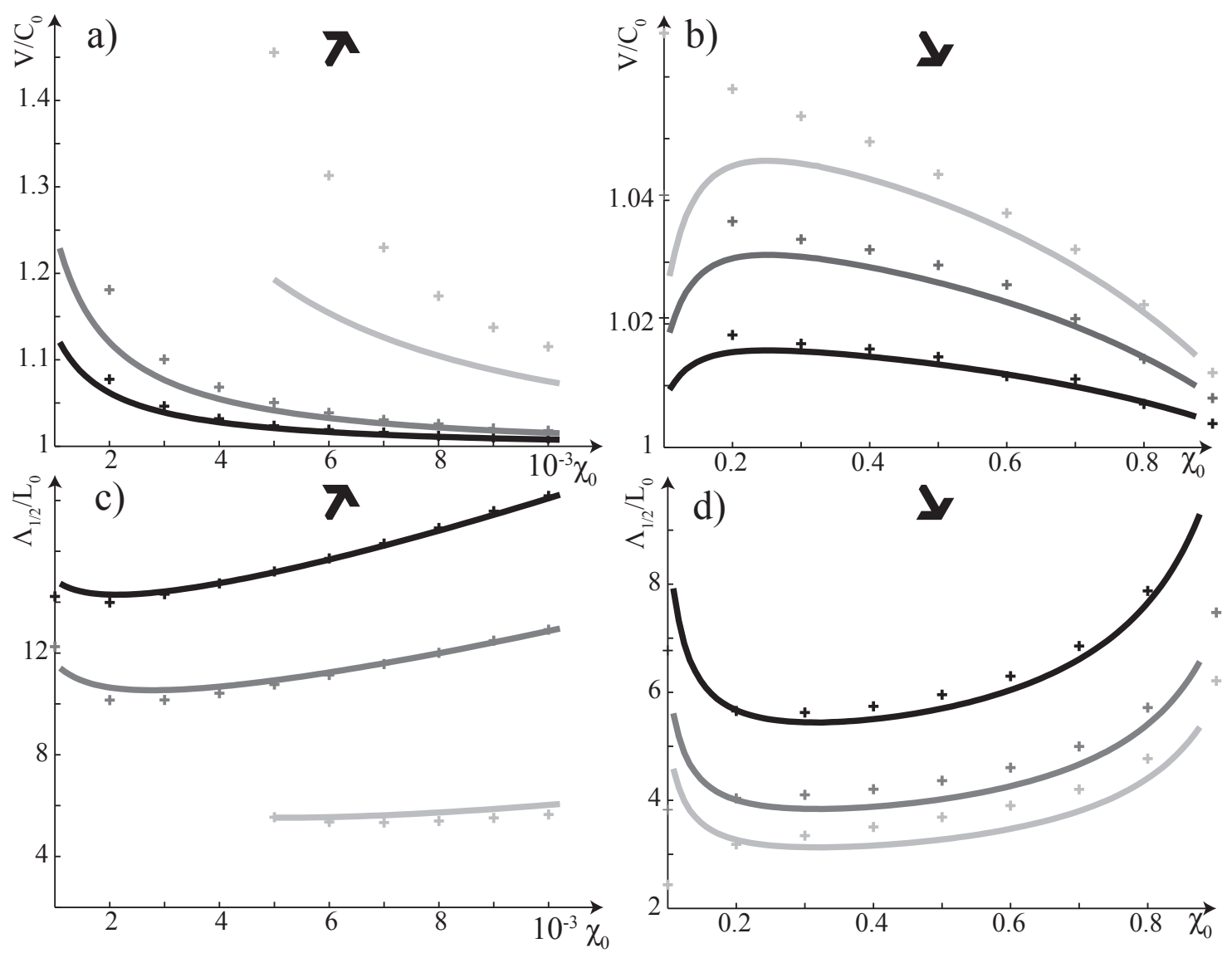

Figure 8: Normalized wave speed $V / C_{0}$ (Eq. 6) (a,b) and normalized width at half amplitude $\Lambda_{1 / 2} / L_{0}$ (Eq. 7 ) $(\mathrm{c}, \mathrm{d})$ in terms of the pre-compression $\chi_{0}$ for the guided-support configuration, computed from the Boussinesq equation (full lines) or from simulations ( + ) with $\Delta \xi_{m}=-\left[\begin{array}{lll}0.5 & 1 & 5\end{array}\right] \times 10^{-3}$ (a,c) and $\Delta \xi_{m}=\left[\begin{array}{lll}0.1 & 0.2 & 0.3\end{array}\right]$ $(\mathrm{b}, \mathrm{d})$ respectively from dark to light gray. Note that no simulations were performed for the highest dynamic amplitude $\left(\Delta \xi_{m}=-5 \times 10^{-3}\right)$ and small buckling level $\left(\chi_{0}<5 \times 10^{-3}\right)$ since the dynamic wave cannot cross the snapping point $\left(\chi_{0}=0\right)$, as shown in Fig. 5a. See Table 1 for arrow notation. 


\subsubsection{Pinned-supported beam}

Simulations for wave propagation in pinned-support configurations using Eq. (60) as input are performed for several levels of compression and snapshots of the strain are shown in Fig. 9.

For $\chi_{0}<\chi_{\sigma}$ (Figs. 9a-c), the input wave is compressive and a small front is present, in agreement with subsonic properties of the pinned-support beam for such level of compression (Table 1). This argument holds also for $\chi_{\sigma}<\chi_{0}<\chi_{\gamma}$ (Figs. 9d,e) where a front is present too with a rarefaction wave. Finally, for $\chi_{0}>\chi_{\gamma}$ (Fig. 9f), the input is in compression, but contrary to Figs. 9a-c, a tail is present, in agreement with the supersonic properties of strongly compressed pinned-support beams (Table 1).

The time required to obtain the asymptotic solution is now investigated. For $\chi_{0}<\chi_{\sigma}$ (Figs. 9a-c), if a front is initially present, its separates quite readily from the main wave, in agreement with the subsonic properties of the solitary wave model. Note now that the number of unit cells compared to the guided-support configuration has been increased by a factor 4 due to the fact that for a given compression $\Delta U_{0}$, the linear speed in the pinnedsupport configuration is 4 times smaller $\left(C_{0}^{2}=P^{\prime}\left\{\Delta U_{0}\right\} L_{0}^{2} / m\right.$ and see $P\left\{\Delta U_{0}\right\}$ curve in Fig. 2). However, for $\chi_{0}>\chi_{\sigma}$ (Figs. 9d-f), despite this extension, the length of the beam is just enough to see the beginning of the wave splitting but it is not immediately clear why the solitary wave takes so long to reach its asymptotic characteristics. In particular, if the wave propagation in the pinned configuration is compared to the one in the guided configuration for the same pre-compression $\chi_{0}=0.9$ (Figs. $7 \mathrm{f}$ and $9 \mathrm{f}$ ), this phenomenon is present only in the case of pinned supports, whereas predicted wave behavior, nonlinearity, and wave amplitude are the same. The dispersion is also almost the same for this level of compression (Fig. 4). Nonlinear coupling between rotations and axial displacement, which is neglected, is weak so that any perturbation would require a large time to separate from the asymptotic solution.

The solitary wave speed and wavelength are plotted in Fig. 10 and compared to the 

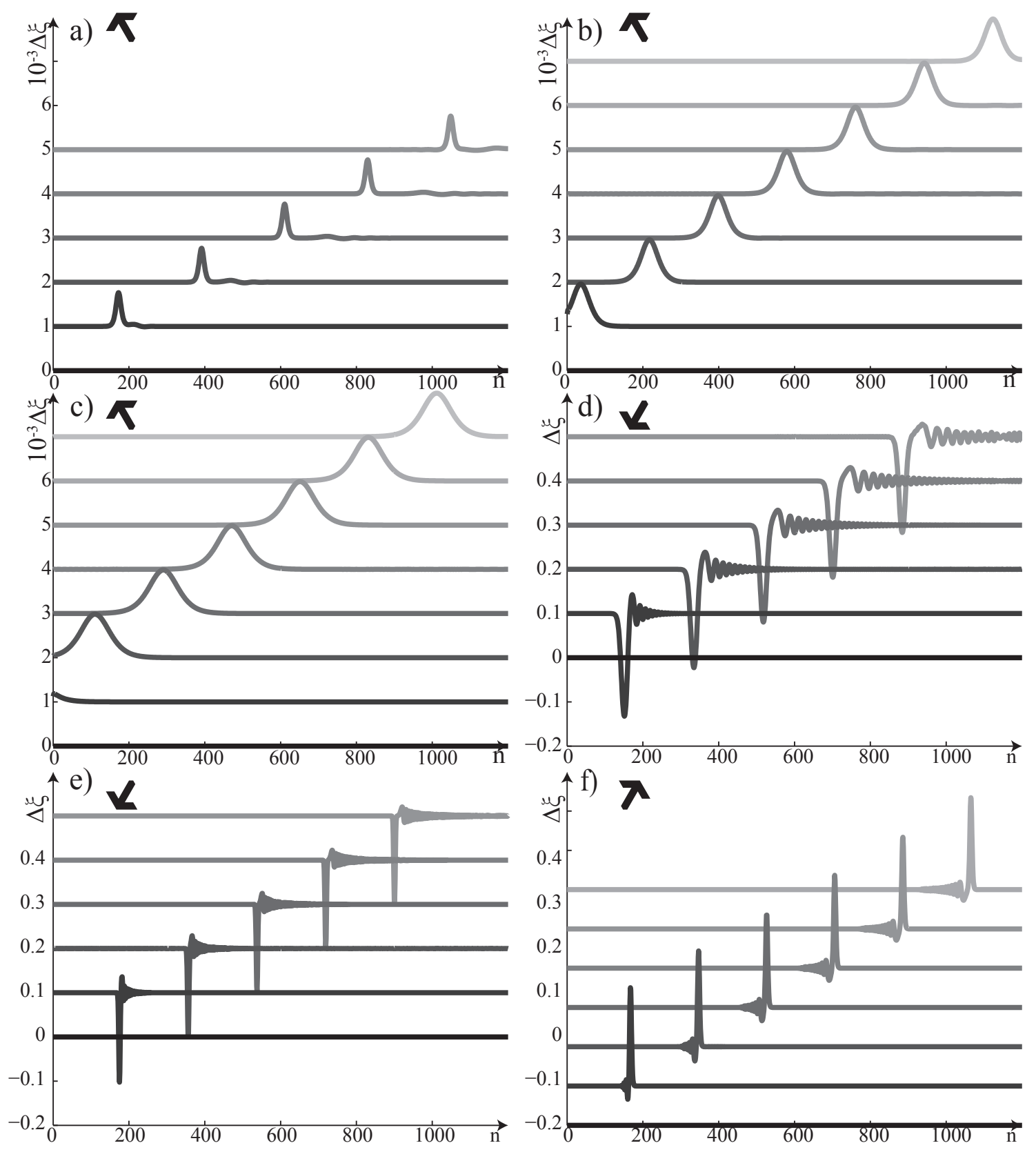

Figure 9: Snapshots at times multiple of $t_{i}=3 / 20 t_{f}$ of the strain wave propagating in the pinned-supported buckled beam with the pre-compression $\chi_{0}=1 \times 10^{-3}$ (a), $\chi_{0}=5 \times 10^{-3}(\mathrm{~b}), \chi_{0}=10 \times 10^{-3}$ (c), $\chi_{0}=0.3$ (d), $\chi_{0}=0.8$ (e) and $\chi_{0}=0.9$ (f) with $\Delta \xi_{m}=1 \times 10^{-3}$ (a,b,c), $\Delta \xi_{m}=-0.3$ (d,e) or $\Delta \xi_{m}=0.3$ (f). See Table 1 for arrow notation.

Boussinesq solution. For $\chi_{0}>\chi_{\gamma}$, the speed increases with the amplitude such that propagating waves are supersonic (Figs. 10b). On the contrary, for $\chi_{0}<\chi_{\gamma}$, the waves are subsonic 
since the speed decreases when the amplitude increases (Figs. 10a,b). Finally, the computed wavelength trend is in agreement with the Boussinesq model although values do not match perfectly (Figs. 10c,d).
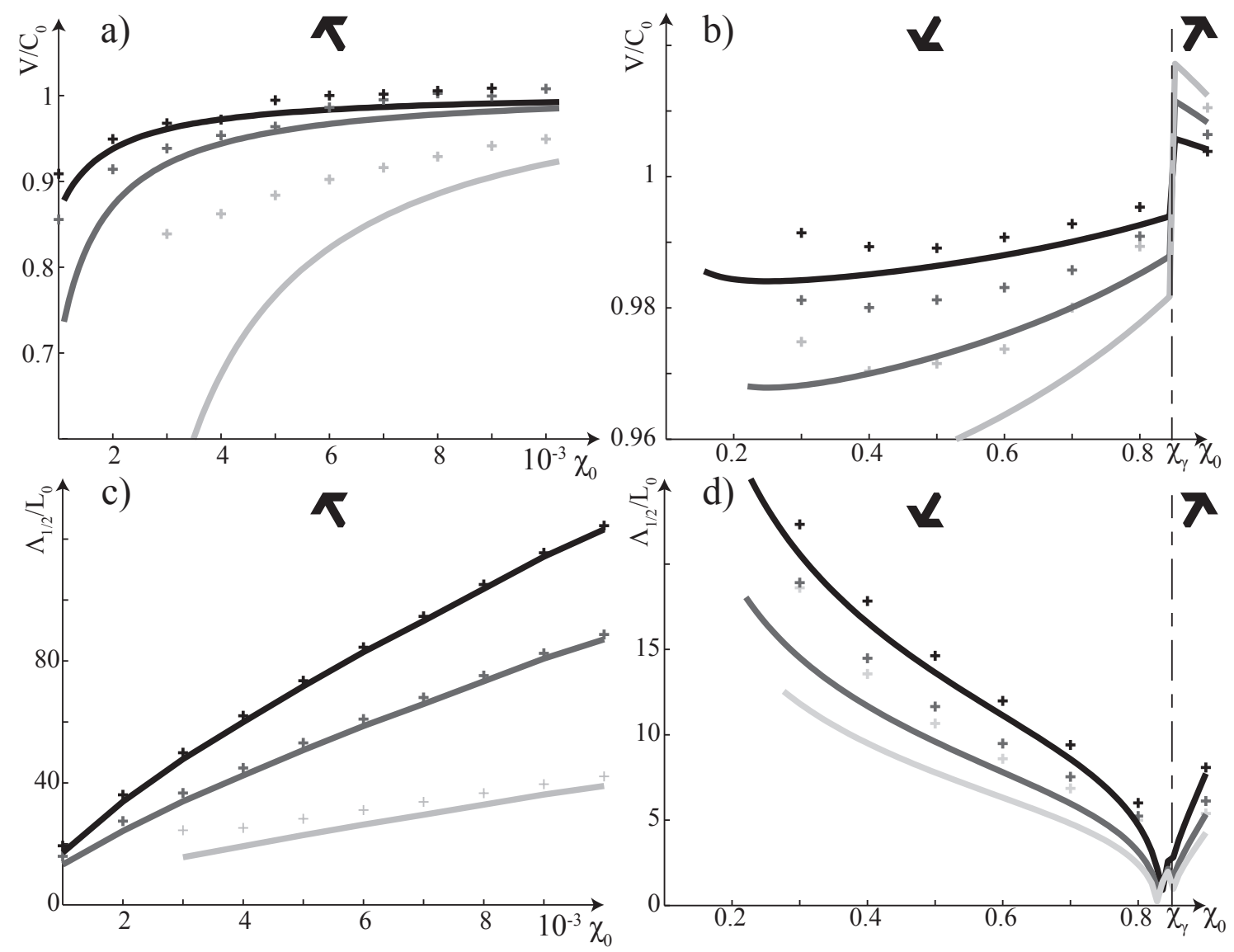

Figure 10: Normalized wave speed $V / C_{0}$ (Eq. 6) (a,b) and normalized width at half amplitude $\Lambda_{1 / 2} / L_{0}$ (Eq. 7 ) $(\mathrm{c}, \mathrm{d})$ in terms of the pre-compression $\chi_{0}$ for the pinned-support configuration, computed from the Boussinesq equation (full lines) or from simulations (+) with $\Delta \xi_{m}=\left[\begin{array}{lll}0.5 & 1 & 5\end{array}\right] \times 10^{-3}(\mathrm{a}, \mathrm{c})$ and $\left|\Delta \xi_{m}\right|=\left[\begin{array}{lll}0.1 & 0.2 & 0.3\end{array}\right]$ $(\mathrm{b}, \mathrm{d})$ respectively from dark to light gray. Note that no simulations were performed for the highest dynamic amplitude $\left(\Delta \xi_{m}=5 \times 10^{-3}\right)$ and small buckling level $\left(\chi_{0}<3 \times 10^{-3}\right)$ since the wave speed cannot be imaginary (Fig. 5b). See Table 1 for arrow notation.

As before, although the trend of the wave speed and wavelength are in agreement with the Boussinesq equation, numerical values are not perfectly recovered specifically when increasing the amplitude for a fixed $\chi_{0}$ or decreasing $\chi_{0}$ for a fixed amplitude. Again this is explained by the fact that the local approximation of the load-displacement relationship by a polynomial 
of degree two is not always appropriate. However, although the input does not correspond directly to the asymptotic solution, the conclusion of this part is that compressive supersonic, rarefaction supersonic, compressive subsonic and rarefaction subsonic solitary waves are obtained.

\subsubsection{Soliton collisions}

Solitary waves that are insensitive to collisions, beyond a phase shift are termed solitons and result from PDEs which are exactly integrable as the Boussinesq equation [40]. Here, wave collision is obtained by exciting the left and right boundary of the structure with waves such that $U_{\text {Right }}=-U_{\text {Left }}$ (Eq. 60). Snapshots of these waves are shown in Fig. 11 for the four different wave types described in Table 1 . Solitary waves are found unchanged by collisions in Figs. 11a-c, whereas for Fig. 11d the presence of a front before the collision discredits direct observations. However, for this last example but not shown in the present paper, a single wave propagating in one direction only will give the same wave as the one propagating in Fig. 11d after the collision.

\subsection{Comparison of the models based on power law and generalized $P\{\Delta U\}$ to FE simulations}

The phase speed in terms of the dynamic amplitude for solitary waves with different levels of compression is shown in Fig. 12. The speed $V$ increases with amplitude, in agreement with supersonic properties of buckled beams with guided supports.

Expectedly, Eq. (59) based on the general relation $P\{\Delta U\}$ provides the best agreement with FE results. Models based on the power-law approximation (Eqs. (47) and (50)) are generally acceptable except for moderated buckling levels, since $P\{\Delta U\}$ is not well approximated in this region (Fig. 2). For the Boussinesq model, the speed $V$ is almost linear in $\Delta \xi_{m}$ $\left(\Delta \xi_{m} \ll 3 C_{0}^{2} / \sigma\right)$ and only the tangent at $V=C_{0}$ is in agreement with FE results. For increasing $\Delta \xi_{m}$, results differ and this is explained again by the fact that the load-displacement relationship cannot be described by a second degree polynomial (Fig. 2). For strong precompression (Fig. 12f), all models are in agreement with FE results. This is explained by the fact that for such level, the dynamic amplitude of the wave relative to the pre-compression 

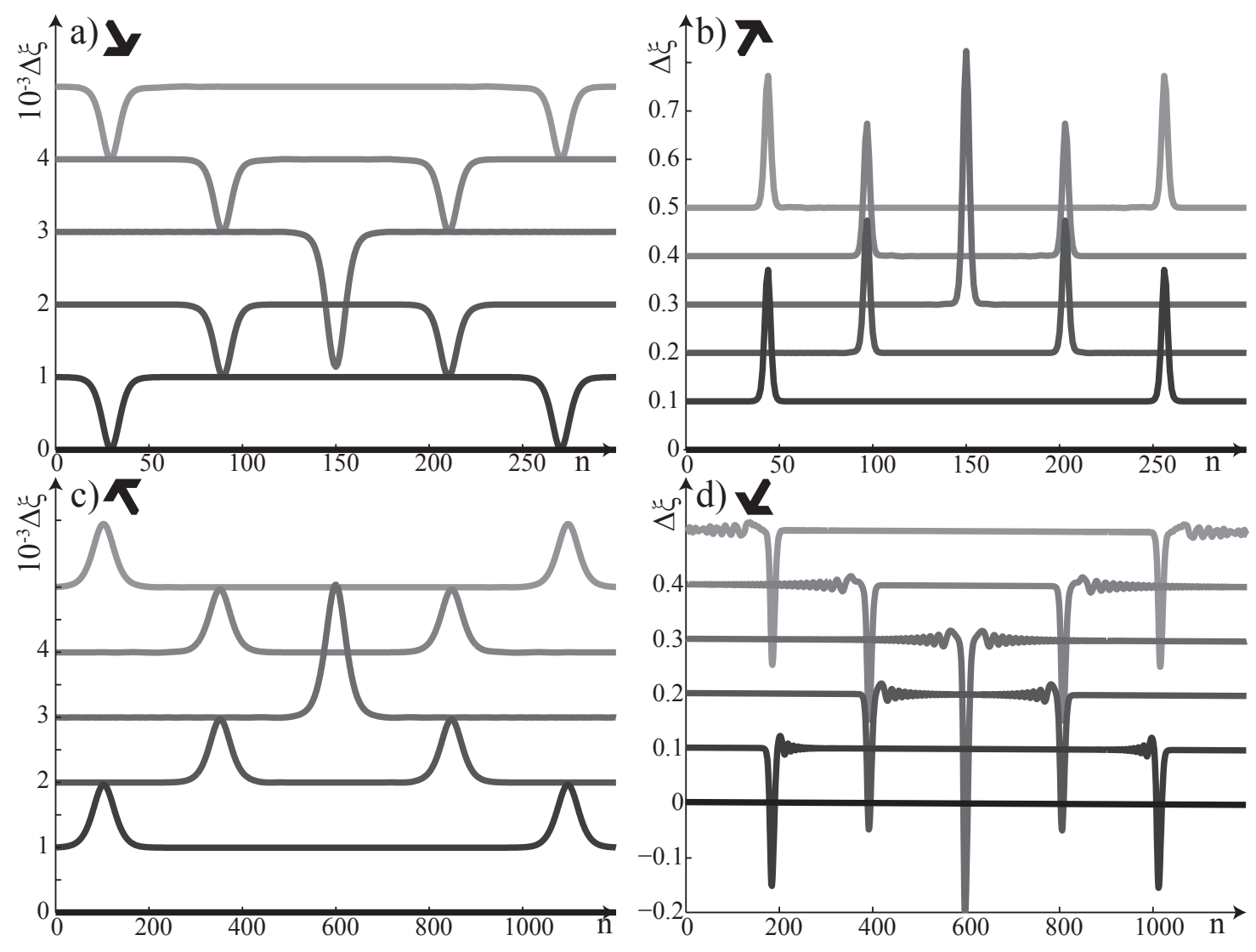

Figure 11: Snapshots of the collision of supersonic rarefaction (a), supersonic compressive (b), rarefaction subsonic (c) and compressive subsonic (d) strain waves given that the pre-compression is $\chi_{0}=5 \times 10^{-3}$ (a,c), $\chi_{0}=10 \times 10^{-3}(\mathrm{~b}, \mathrm{~d})$, and the dynamic amplitude is $\left|\Delta \xi_{m}\right|=1 \times 10^{-3}(\mathrm{a}, \mathrm{c})$ and $\left[\Delta \xi_{m}\right]=0.3(\mathrm{~b}, \mathrm{~d})$. Time of snapshots is in multiples of $0.1137 \mathrm{~s}$ (a), $0.0736 \mathrm{~s}$ (b), $1.0403 \mathrm{~s}$ (c) and $1.4613 \mathrm{~s}$ (d) such that the fourth snapshot corresponds to the time with the maximum amplitude. See Table 1 for arrow notation.

strain is small, justifying Eq. (2) or Eq. (33). Finally, note that the two models based on the power law (Eq. (38) and (39)) or the two models based on the general function (Eq. (56) and (57)) are similar validating the assumption to neglect the last terms of Eqs. (38), (39), (56) and (57). Since both models are similar, the ratio $\left|a_{2,2} / a_{0,4}\right|$ and so the choice of the model to use is not significant. Results for the case of pinned supports are not given since they provide similar conclusions.

Wavelength is less sensitive to $\Delta \xi_{m}$ (Fig. 13) compared to the speed (Fig. 12). Indeed, both the Boussinesq and the power-law models disagree with FE simulations only for the case of $\chi_{0}=0.1$ (Fig. 13d), and this is explained by the fact that $\lambda$ is mostly determined by 

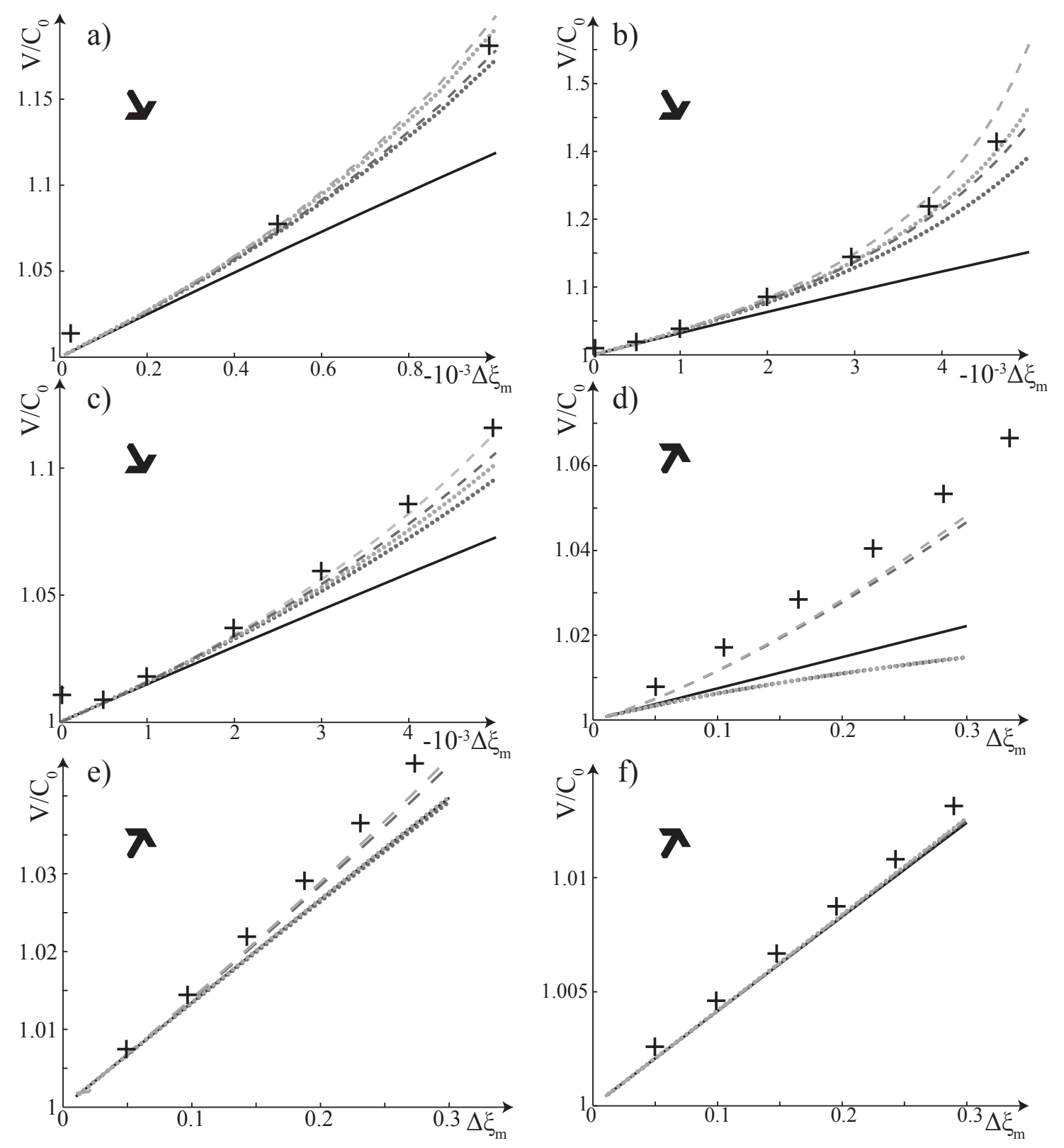

Figure 12: Normalized solitary wave speed $V / C_{0}$ in terms of the wave amplitude $\left|\Delta \xi_{m}\right|$ for the guided-support configuration with pre-compression $\chi_{0}=2 \times 10^{-3}$ (a), $\chi_{0}=6 \times 10^{-3}$ (b), $\chi_{0}=10 \times 10^{-3}$ (c), $\chi_{0}=0.1$ (d), $\chi_{0}=0.5$ (e) and $\chi_{0}=0.9$ (f). Results from FE simulations with (+), improved Boussinesq model Eq. (6) in full dark lines, power law with Eq. (38) in dark-gray dotted lines, power law with Eq. (39) in light-gray dotted lines, general function with Eq. (56) in dark-gray dashed lines and general function with Eq. (57) in light-gray dashed lines. See Table 1 for arrow notation.

$C_{0}$. 


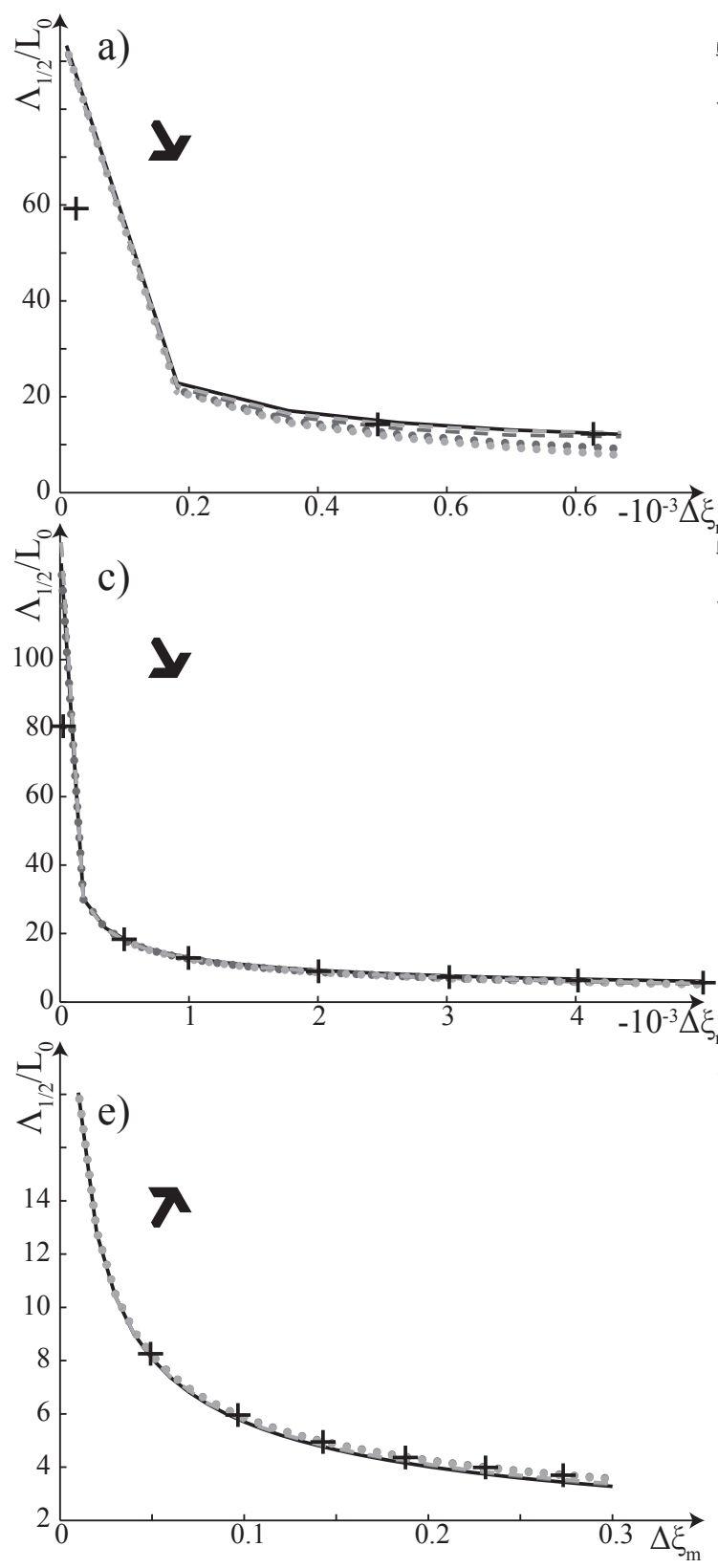

b)
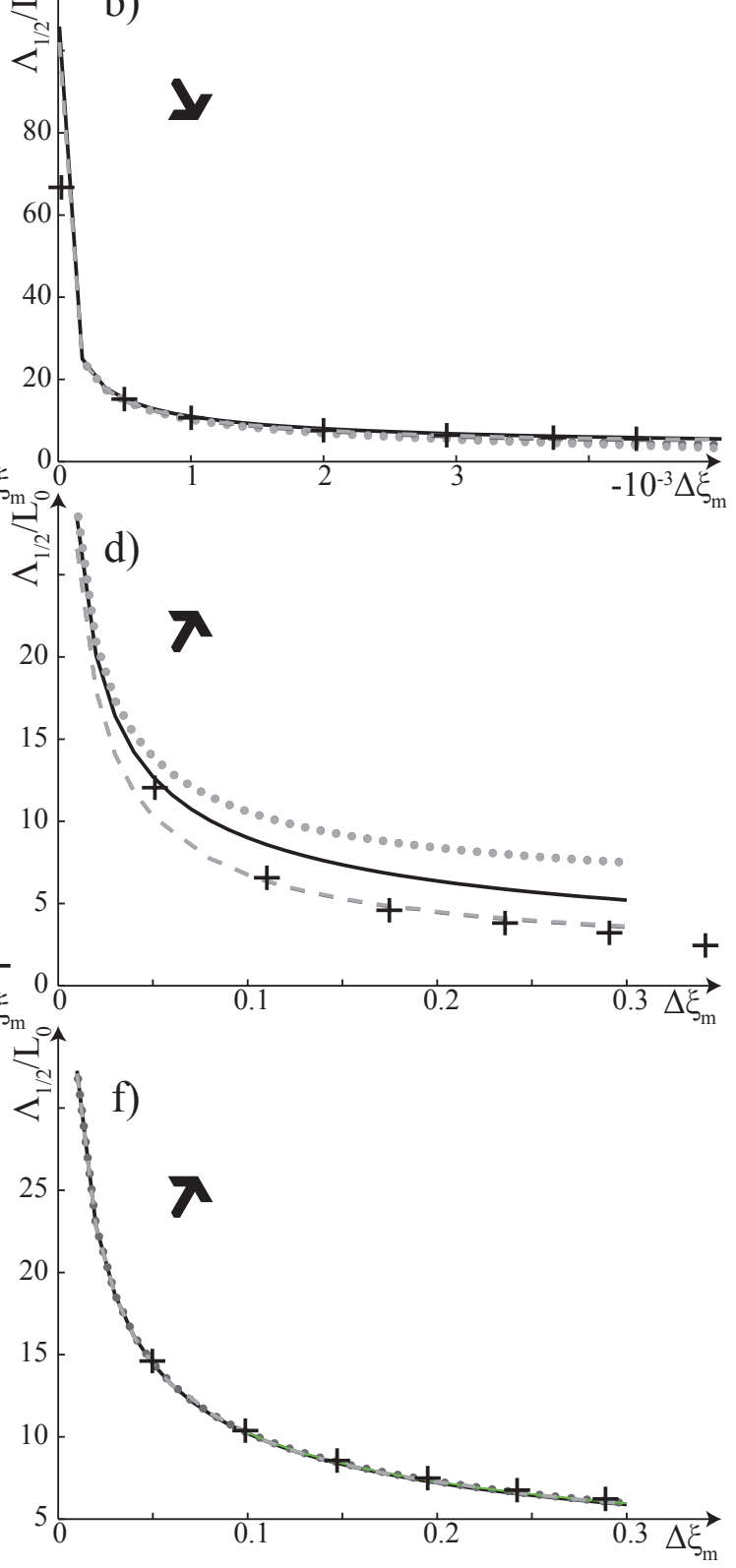

Figure 13: Normalized solitary wavelength $\Lambda_{1 / 2} / L_{0}$ in terms of the wave amplitude $\left|\Delta \xi_{m}\right|$ for the guidedsupport configuration with pre-compression $\chi_{0}=2 \times 10^{-3}$ (a), $\chi_{0}=6 \times 10^{-3}$ (b), $\chi_{0}=10 \times 10^{-3}$ (c), $\chi_{0}=0.1(\mathrm{~d}), \chi_{0}=0.5(\mathrm{e})$ and $\chi_{0}=0.9$ (f). Results from FE simulations with (+), improved Boussinesq model Eq. (6) in full dark lines, power law with Eq. (38) in dark-gray dotted lines, power law with Eq. (39) in light-gray dotted lines, general function with Eq. (56) in dark-gray dashed lines and general function with Eq. (57) in light-gray dashed lines. See Table 1 for arrow notation. 


\section{Conclusions}

Wave propagation in buckled beams is investigated and depending on the pre-compression and dynamic amplitude, various homogenized PDEs describe the propagation of stable, nonlinear waves are found. Where the load-deformation can be described by a polynomial of degree two, the Boussinesq equation can be employed. Even though the fidelity of predicted velocity and wavelength depend on appropriate representation of the load-displacement relation, the pre-compression level and support type furthermore determine dispersion characteristics of the resulting medium. This leads to the Boussinesq equation, from which it is shown that four types of solitary waves are possible. Namely combination of subsonic, supersonic, compressive and rarefaction waves. Where power-law and general load-displacement relations are required, strongly nonlinear PDEs results from homogenization. Here, existing techniques are extended for solitary-wave solutions based on equivalent potentials. Finite element simulations validate analytical models. In conclusion, a qualitative prediction for the type of propagating wave, its speed and shape, various forms of the Boussinesq equation are the simplest model to implement and use. Homogenized PDEs based on the exact load-displacement relation provide best results but require numerical integration. For small buckling level and guided-supports, the model based on power-law nonlinearities is also a good alternative.

\section{Acknowledgment}

The authors acknowledge the Swiss National Science Foundation (SNSF) for supporting this work with project 200021137894.

\section{Appendix A.}

In order to justify the assumption of a quasi-static load-deformation model of the unit cell, it is shown here that the frequency content of the Boussinesq solution do no excite resonant frequencies of the structure. The magnitude of the frequency spectrum $|X(f)|$ of the function $f=\operatorname{sech}^{2}(t / T)$ is plotted in Fig. A.1b, and most of the frequency components are found 
bellow the frequency $1 / T$. Moreover, the first resonant frequency, corresponding to the first band-gap in the dispersion curve, is obtained using the Bloch theorem applied to a discrete glide-periodic unit cell [42].

When the frequency spectrums of the soliton solutions are compared to the first resonance frequency of the structure, they are found at least two orders of magnitude smaller (see Figs. A.1c,d), justifying the restriction of the wave propagation analysis to the low-frequency regime.
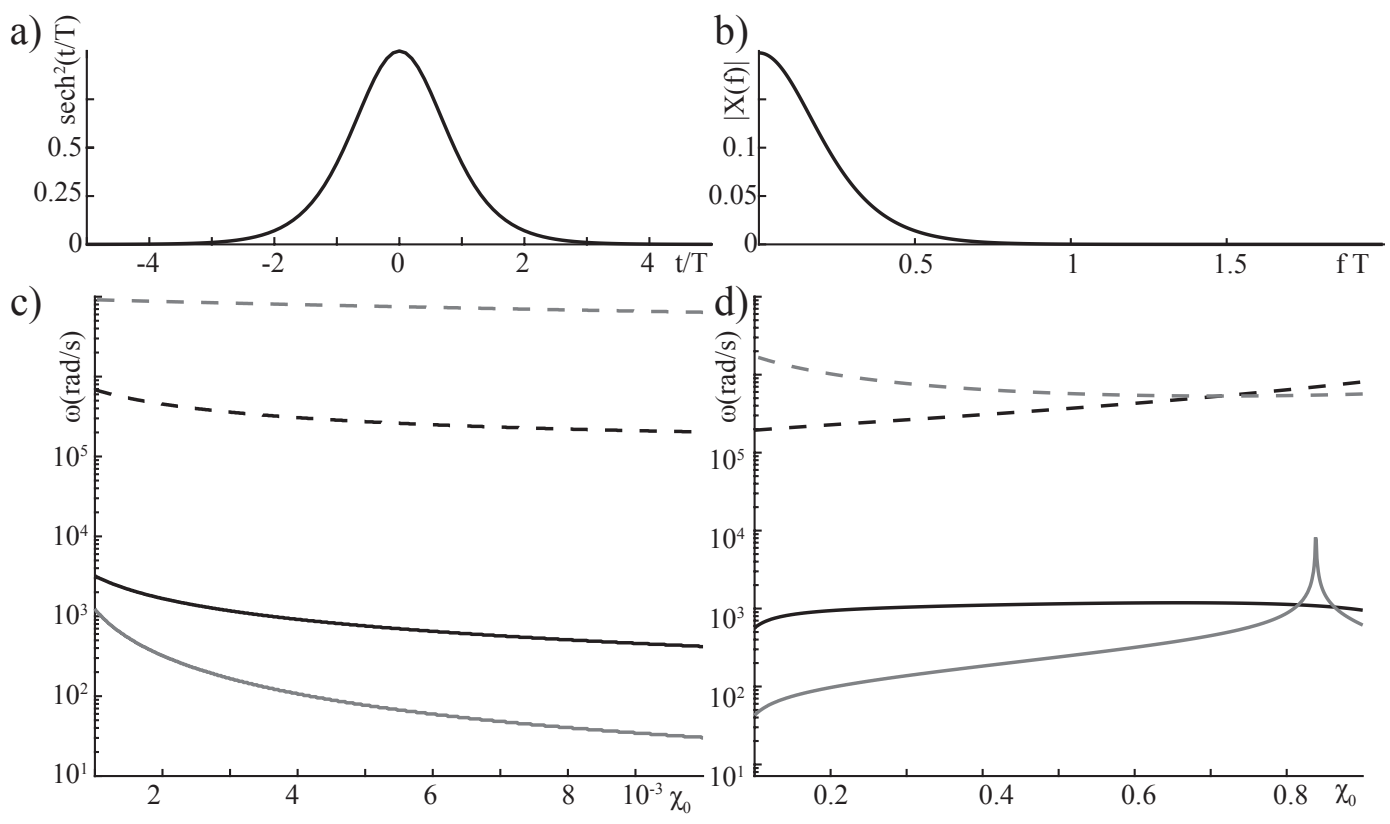

Figure A.1: The function $f=\operatorname{sech}^{2}(t / T)$ (a) and its frequency spectrum $|X(f)|$ (b). Resonant frequency of the structure (dashed lines) and maximum frequency content of the function $f$ (full lines), where $T=\Lambda / V$ is computed from Eqs. (6) and (7) with $\Delta \xi_{m}=-1 \times 10^{-3}$ (c) and $\Delta \xi_{m}=0.2$ (d). Results are shown in terms of the initial compression $\chi_{0}$, and the guided and pinned support configurations are respectively in black and gray lines $(\mathrm{c}, \mathrm{d})$.

[1] F. Maurin, A. Spadoni, Low-frequency wave propagation in post-buckled structures, Wave Motion 51 (2) (2014) 323-334.

[2] M. Remoissenet, Waves Called Solitons: Concepts and Experiments, Vol. 63, Springer, 1995. 
[3] L. Munteanu, S. Donescu, Introduction to soliton theory: applications to mechanics, Kluwer Academic Publishers, 2004.

[4] E. Kim, F. Li, C. Chong, G. Theocharis, J. Yang, P. G. Kevrekidis, Highly Nonlinear Wave Propagation in Elastic Woodpile Periodic Structures, Physical Review Letters 114 (11) (2015) 118002.

[5] F. Fraternali, L. Senatore, C. Daraio, Solitary waves on tensegrity lattices, Journal of the Mechanics and Physics of Solids 60 (6) (2012) 1137-1144.

[6] F. Fraternali, G. Carpentieri, A. Amendola, R. E. Skelton, V. F. Nesterenko, Multiscale tunability of solitary wave dynamics in tensegrity metamaterials, Applied Physics Letters 105 (20) (2014) 201903.

[7] H. Yasuda, C. Chong, E. G. Charalampidis, P. G. Kevrekidis, J. Yang, Formation of rarefaction waves in origami-based metamaterials, arXiv preprintarXiv:1505.03752.

[8] G. A. Maugin, Nonlinear Waves in Elastic Crystals, Oxford University Press, 1999.

[9] K. R. Khusnutdinova, A. M. Samsonov, A. S. Zakharov, Nonlinear layered lattice model and generalized solitary waves in imperfectly bonded structures, Physical Review E Statistical, Nonlinear, and Soft Matter Physics 79 (5) (2009) 056606.

[10] A. V. Porubov, I. E. Berinskii, Non-linear plane waves in materials having hexagonal internal structure, International Journal of Non-Linear Mechanics 67 (2014) 27-33.

[11] V. F. Nesterenko, Dynamics of Heterogeneous Materials, Springer, 2001.

[12] S. Sen, J. Hong, J. Bang, E. Avalos, R. Doney, Solitary waves in the granular chain, Physics Reports 462 (2) (2008) 21-66.

[13] M. Destrade, G. Saccomandi, Solitary and compactlike shear waves in the bulk of solids., Physical review. E, Statistical, nonlinear, and soft matter physics 73 (6 Pt 2) (2006) 065604. 
[14] M. Destrade, G. Saccomandi, Nonlinear transverse waves in deformed dispersive solids, Wave Motion 45 (3) (2008) 325-336.

[15] B. D. Coleman, E. H. Dill, D. Swigon, On the dynamics of flexure and stretch in the theory of elastic rods, Archive for Rational Mechanics and Analysis 129 (2) (1995) $147-174$.

[16] A. R. Champneys, G. W. Hunt, J. M. T. Thompson, Localization and solitary waves in solid mechanics, World Scientific, 1997.

[17] A. M. Samsonov, G. A. Maugin, Strain Solitons in Solids and How to Construct Them, Vol. 54, CRC Press, 2001.

[18] A. V. Porubov, Amplification of nonlinear strain waves in solids, World Scientific, 2003.

[19] A. V. Porubov, G. A. Maugin, Longitudinal strain solitary waves in presence of cubic non-linearity, International Journal of Non-Linear Mechanics 40 (7) (2005) 1041-1048.

[20] B. Duričković, A. Goriely, G. Saccomandi, Compact waves on planar elastic rods, International Journal of Non-Linear Mechanics 44 (5) (2009) 538-544.

[21] J. Genzer, J. Groenewold, Soft matter with hard skin: From skin wrinkles to templating and material characterization, Soft Matter 2 (4) (2006) 310.

[22] X. Chen, J. W. Hutchinson, A family of herringbone patterns in thin films, Scripta Materialia 50 (6) (2004) 797-801.

[23] A. Takei, F. Brau, B. Roman, J. Bico, Stretch-induced wrinkles in reinforced membranes: From out-of-plane to in-plane structures, EPL (Europhysics Letters) 96 (6) (2011) 64001.

[24] A. Goriely, R. Vandiver, M. Destrade, Nonlinear Euler buckling, Proceedings of the Royal Society A: Mathematical, Physical and Engineering Sciences 464 (2099) (2008) 3003-3019. 
[25] J. Xiong, R. Ghosh, L. Ma, A. Vaziri, Y. Wang, L. Wu, Sandwich-walled cylindrical shells with lightweight metallic lattice truss cores and carbon fiber-reinforced composite face sheets, Composites Part A: Applied Science and Manufacturing 56 (2014) 226-238.

[26] A. Vaziri, On the buckling of cracked composite cylindrical shells under axial compression, Composite Structures 80 (1) (2007) 152-158.

[27] K. Sad Saoud, P. Le Grognec, A unified formulation for the biaxial local and global buckling analysis of sandwich panels, Thin-Walled Structures 82 (0) (2014) 13-23.

[28] L. Léotoing, S. Drapier, a. Vautrin, First applications of a novel unified model for global and local buckling of sandwich columns, European Journal of Mechanics, A/Solids 21 (4) (2002) 683-701.

[29] B. Audoly, Y. Pomeau, Elasticity and Geometry: From hair curls to the non-linear response of shells, Vol. 2, OUP Oxford, 2010.

[30] J. T. B. Overvelde, S. Shan, K. Bertoldi, Compaction through buckling in 2D periodic, soft and porous structures: effect of pore shape., Advanced materials (Deerfield Beach, Fla.) 24 (17) (2012) 2337-42.

[31] S. H. Kang, S. Shan, A. Košmrlj, W. L. Noorduin, S. Shian, J. C. Weaver, D. R. Clarke, K. Bertoldi, Complex Ordered Patterns in Mechanical Instability Induced Geometrically Frustrated Triangular Cellular Structures, Physical Review Letters 112 (9) (2014) 098701.

[32] B. Haghpanah, J. Papadopoulos, D. Mousanezhad, H. Nayeb-Hashemi, A. Vaziri, Buckling of regular, chiral and hierarchical honeycombs under a general macroscopic stress state, Proceedings of the Royal Society A: Mathematical, Physical and Engineering Sciences 470 (2167) (2014) 20130856. 
[33] Y. Sun, W. M. Choi, H. Jiang, Y. Y. Huang, J. A. Rogers, Controlled buckling of semiconductor nanoribbons for stretchable electronics., Nature nanotechnology 1 (3) (2006) 201-207.

[34] F. Brau, H. Vandeparre, A. Sabbah, C. Poulard, A. Boudaoud, P. Damman, Multiplelength-scale elastic instability mimics parametric resonance of nonlinear oscillators, Nature Physics 7 (1) (2010) 56-60.

[35] S. Cai, D. Breid, A. Crosby, Z. Suo, J. Hutchinson, Periodic patterns and energy states of buckled films on compliant substrates, Journal of the Mechanics and Physics of Solids 59 (5) (2011) 1094-1114.

[36] F. Maurin, A. Spadoni, Wave dispersion in periodic post-buckled structures, Journal of Sound and Vibration 333 (19) (2014) 4562-4578.

[37] G. Trainiti, J. Rimoli, M. Ruzzene, Wave propagation in periodically undulated beams and plates, International Journal of Solids and Structures 75-76 (2015) 260-276.

[38] F. Maurin, A. Spadoni, Wave Propagation in Periodic Buckled Beams. Part II: Experiments, Submitted to Wave Motion.

[39] Z. P. Bazant, L. Cedolin, J. W. Hutchinson, Stability of Structures: Elastic, Inelastic, Fracture, and Damage Theories, Vol. 60, World Scientific, 1993.

[40] M. J. Ablowitz, Nonlinear Dispersive Waves: Asymptotic Analysis and Solitons, Cambridge University Press, 2011.

[41] W. R. D. Cook, D. S. Malkus, Concepts and applications of finite element analysis, Wiley India Pvt. Limited, 2002.

[42] F. Maurin, Bloch theorem with revised boundary conditions applied to glide and screw symmetric, quasi-one-dimensional structures, Wave Motion 61 (2016) 20-39. 
[43] P. P. Delsanto, Universality of Nonclassical Nonlinearity, Springer Science \& Business Media, 2006.

[44] A. Berezovski, J. Engelbrecht, A. Salupere, K. Tamm, T. Peets, M. Berezovski, Dispersive waves in microstructured solids, International Journal of Solids and Structures 50 (11-12) (2013) 1981-1990.

[45] D. Sun, C. Daraio, S. Sen, Nonlinear repulsive force between two solids with axial symmetry, Physical Review E - Statistical, Nonlinear, and Soft Matter Physics 83 (6) (2011) 066605.

[46] J. M. Battini, Co-rotational beam elements, Tech. rep., Royal Institute of Technology (2002).

[47] F. Maurin, L. Dedè, A. Spadoni, Isogeometric rotation-free analysis of planar extensibleelastica for static and dynamic applications, Nonlinear Dynamics.

[48] J. Chung, G. M. Hulbert, A Time Integration Algorithm for Structural Dynamics With Improved Numerical Dissipation: The Generalized- $\alpha$ Method, Journal of Applied Mechanics 60 (2) (1993) 371.

[49] S. B. Raknes, X. Deng, Y. Bazilevs, D. J. Benson, K. M. Mathisen, T. Kvamsdal, Isogeometric rotation-free bending-stabilized cables: Statics, dynamics, bending strips and coupling with shells, Computer Methods in Applied Mechanics and Engineering 263 (2013) 127-143. 